\title{
The prevalence and the impact of sarcopenia in digestive cancers. A systematic review
}

\author{
CARMEN HAIDUCU ${ }^{1}$, ADRIAN BUZEA ${ }^{1,2}$, LILIANA ELENA MIREA $^{1,3}$, GHEORGHE ANDREI DAN $^{1,2}$ \\ ${ }^{1}$ Carol Davila University of Medicine and Pharmacy, Bucharest, Romania. \\ ${ }^{2}$ Cardiology, Clinical Hospital Colentina, Bucharest, Romania \\ ${ }^{3}$ Anesthesia and Intensive Care Unit, Emergency Clinical Hospital, Bucharest, Romania
}

\begin{abstract}
Introduction: Sarcopenia is characterized by a decrease in skeletal muscle mass, associated with low muscle strength and/or poor physical performance. Assessing the prevalence of sarcopenia among digestive cancers and establishing the impact that sarcopenia has on the postoperative evolution of digestive tumors may be a central pillar in improving postoperative outcomes by caring for perioperative sarcopenia.

This brief review aimed to evaluate the prevalence of sarcopenia in digestive cancer patients.

Method and materials: PubMed database was searched for "sarcopenia" AND "digestive cancers" from January 1st, 2010, through September 30th, 2020. PRISMA guideline was used for this systematic review. After the selection process, 31 complete studies were included in our review.

Assessment of sarcopenia diagnosis for the studies included in this systematic review was based on a computed tomographic calculation of the skeletal muscle index at the third lumbar vertebra.

Results: Among a total of 11,651 patients with digestive cancers, the prevalence of sarcopenia was $43.68 \%$.

The highest prevalence of sarcopenic patients was in esophageal $(70.4 \%)$ and hepatic $(60.3 \%)$ cancer, following by biliary tract $(49.3 \%)$, pancreatic $(45.70 \%)$, colorectal $(42.83 \%)$ cancer, and gastric cancer $(32.05 \%)$ with the lowest prevalence.

The results of the studies conducted by now regarding the prevalence of sarcopenia in digestive cancers and its relevance in the evolution of these cancers are discordant and uneven.

Some studies show that the presence of sarcopenia in patients with digestive cancers is associated with an increased rate of postoperative complications, increased toxicity of chemotherapeutics and increased mortality. Other studies do not find sarcopenia as an independent risk factor associated with negative consequences in the course of patients with digestive cancers.

Conclusions: Sarcopenia is prevalent in digestive cancers. There is still no consensus about the impact of sarcopenia on the treatment of digestive cancers. Further studies are needed to evaluate the real consequences of sarcopenia in digestive cancers..
\end{abstract} muscles.

Key words: sarcopenia, prevalence, tomography, malnutrition, chemotherapy, abdominal

\section{INTRODUCTION}

According to the updated definition of the European Working Group on Sarcopenia in Older People (EWGSOP), sarcopenia is a muscle disease defined as a progressive decrease in the quality and quantity of skeletal muscle and deterioration of physical function and performance [1]. Sarcopenia is probable when low muscle strength is detected, but the diagnosis is confirmed by the presence of low muscle quantity or quality. When low muscle strength, low muscle quantity/quality, and low physical performance are all detected, sarcopenia is considered severe [1].

The loss of muscle mass and strength accompanying aging is called primary sarcopenia and could be regarded as a normal and physiological phenomenon. Sarcopenia which occurs and worsens concomitantly with oncologic and inflammatory systemic diseases, some type of chronic diseases, or following polytrauma is considered secondary sarcopenia (SeS).

Among the first who analyzed the primary sarcopenia was Mcdonald Critchley, a neurologist at Kings College Hospital in London, in 1931. In 1970, Nathan Shock published a series of articles about this topic. The term sarcopenia was suggested by Irwin Rosenberg at a meeting in Albuquerque, New Mexico, in 1988 [2].

Between 1979 and 1981, Heymsfield and collab. were the first who reported the use of 
computed tomography (CT) images to evaluate skeletal muscle mass and adipose tissue. [3] In 2008, Prado et al. showed that a low L3 muscle index was associated with muscular impairment in patients with malignant disorders of the upper respiratory tract and digestive tract [4].

The recommended method as a "gold standard" for the diagnostic of sarcopenia in digestive cancers is the calculated skeletal muscle index (SMI) by computed tomography method $[1,5]$. Using specialized software, the appendicular skeletal muscle mass (ASM) at lumbar L3/L4 is calculated, then SMI, using the formula ASM/height ${ }^{2}$. The cutoff points for men and women are: ASM $<20 \mathrm{~kg}$, and respectively $15 \mathrm{~kg}$ and $\mathrm{SMI}<7.0 \mathrm{~kg} / \mathrm{m}^{2}$, and respectively $<5.5 \mathrm{~kg} / \mathrm{m}^{2}[1]$.

Skeletal muscle mass (SMM) is accounting for 30-40\% of total body weight [6], [7]. Approximately $75 \%$ of SMM is located in the appendicular region [8].

The appendicular skeleton includes the bones of the shoulder belt, the upper limbs, the pelvic belt, and the lower limbs [9]. Reduction of appendicular skeletal muscle mass (ASMM ) as a surrogate for sarcopenia is accompanied by negative health consequences such as weakness, disability, deteriorated quality of life (QOL), and mortality resulting in increased health care costs [10].

Using CT evaluation at lumbar L3, Prado et al. proposed for the diagnosis of sarcopenia a new formula based on the skeletal muscle area (SMA), which correlates with the whole-body skeletal muscle mass. The formula used is $\mathrm{SMI}=\mathrm{SMA} /$ height $^{2}$ and the normal values are $\leq 52.4 \mathrm{~cm}^{2} / \mathrm{m}^{2}$ for males, and $\leq 38.5 \mathrm{~cm}^{2} / \mathrm{m}^{2}$ for females [4].

Dual-energy x-ray absorptiometry (DXA) are used to measure the SMM with high accuracy, but are expensive and not widely accessible and usually have high radiation exposure [11].

In recent years interest in sarcopenia associated with cancers has increased. As proof, the number of publications on the topic of secondary sarcopenia increased progressively, from 5 in 2010 to 61 papers in 2019 and 46 in 2020 [12].

Considering the increased prevalence of sarcopenia among digestive cancers [4,5], and the negative impact of preoperative sarcopenia on the postoperative outcomes in gastrointestinal cancers [13,14], a guideline for the diagnosis and management of sarcopenia in these patients may be useful.

The present systematic review aims to analyze the prevalence of sarcopenia in patients with digestive cancers.
This systematic review intended to sumarize the literature sparse data on the prevalence of sarcopenia in digestive cancers, without being limited to a specific segment of the digestive tract or organ and whithout being limited by stage disease.

\section{MATERIALS AND METHODS}

For this systematic review, the Preferred Reporting Items for Systematic Reviews and MetaAnalyses (PRISMA) guideline was used [15].

\section{Search strategy}

We performed a systematic literature search on PubMed, Web of Knowledge, and Scopus databases from January 2010 to September 2020. The last search was on 30th September 2020.

The search query terms were: "sarcopenia" [MeSH Terms] OR" sarcopenia" [All Fields] AND "neoplasms" [MeSH Terms] OR "neoplasms" [All Fields] OR "cancers" [All Fields]/ AND "gastrointestinal neoplasms" [MeSH Terms] OR "gastrointestinal neoplasms" [All Fields]/ AND "gastrointestinal cancer" [MeSH Terms] OR "gastrointestinal cancers" [All Fields]/AND "pancreatic cancer/hepatic cancer/cholangiocarcinoma" [MeSH Terms] OR "pancreatic cancer/hepatic cancer/cholangiocarcinoma" [All Fields].

We considered for inclusion the full-text publications, in English. We subsequently included articles identified in other reviews, which were not found by the search strategy.

\section{Eligibility criteria}

The main criteria used for selecting the articles were: 1 . reporting the prevalence of sarcopenia 2. the diagnosis of sarcopenia to be assessed by computed tomography method 3. a definite diagnosis of a gastrointestinal neoplasm (esophageal, gastric, colorectal, pancreatic, hepatic, or cholangiocarcinoma) of any stage, according to the TNM classification.

For the diagnosis of sarcopenia, we considered the computed tomography at the level of the third lumbar vertebra (L3) method and the calculated SMI based on total abdominal muscle area (TAMA), total psoas area (TPA), skeletal muscle mass (SMM), or appendicular skeletal muscle mass (ASM) adjusted by height. The cut-off values for the SMI were considered those recommended by the European Working Group on Sarcopenia in Older People (EWGSOP)[[1]] and the Asian Working Group for Sarcopenia (AWGS) [16]. 
We excluded the publications reporting the prevalence of secondary sarcopenia using other methods different from CT scan at the level of L3 vertebra.

Two authors (CH and LEM) independently analyzed the studies for eligibility. The differences were discussed with other authors until a consensus was reached.

\section{Data collection}

For each study included we collected the data regarding the type of study and publication year, the population (number of patients, sex, mean age), the prevalence, and method of assessment of sarcopenia, the type of cancer, the distribution of patients on the different types of cancer, data regarding morbidity and mortality.

\section{Quality assessment}

The Newcastle-Ottawa assessment scale for case-control studies was used to assess the study quality [17]. The studies were classified as low quality (score $<5$ points), fair quality (6-7 points), and good quality 8-9 points) (Figure 1).

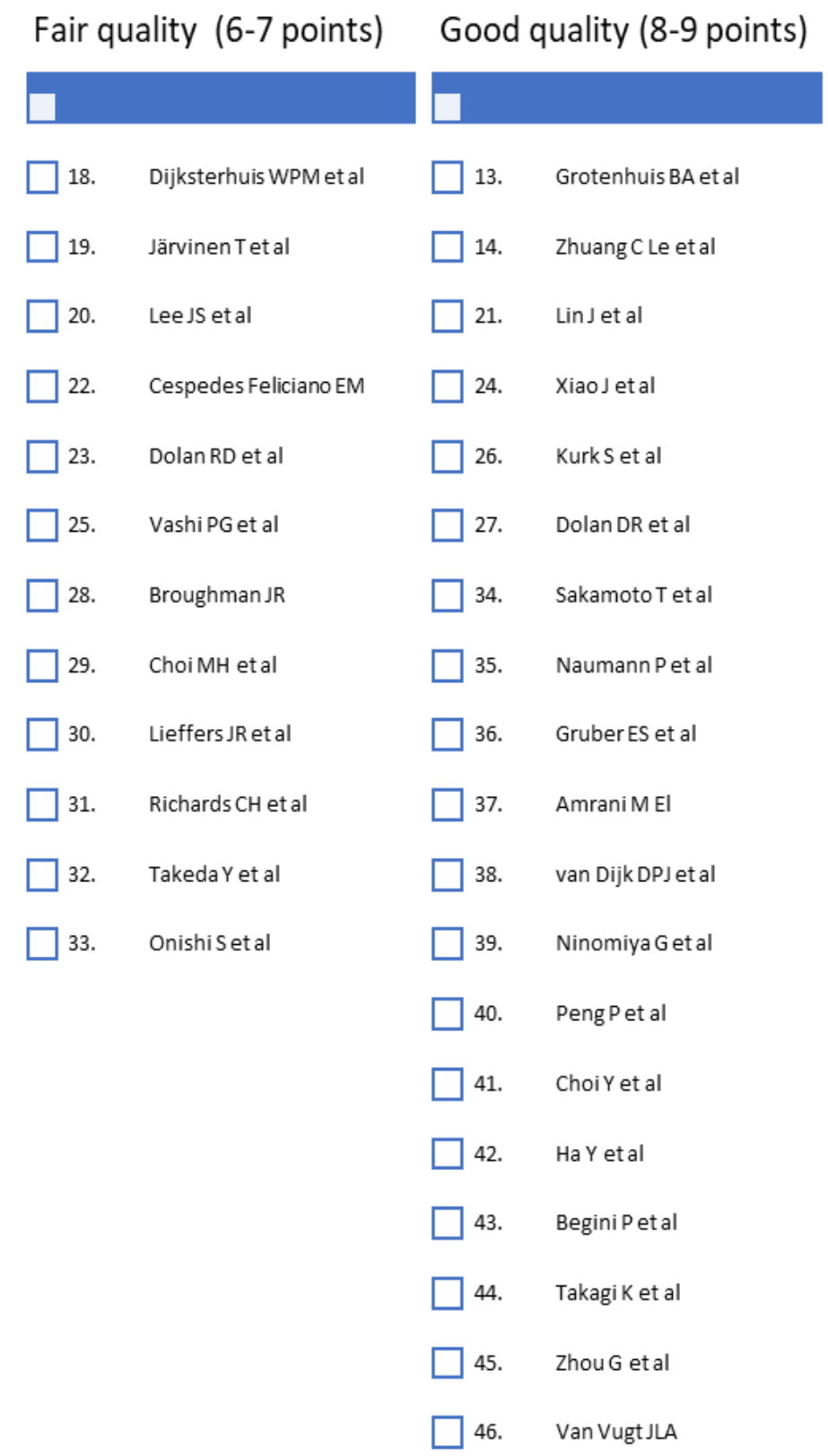

Figure 1. Quality assesment scale chart. 


\section{RESULTS}

251 papers were identified through database searching. After screening of the title and abstract, 76 full-text articles were considered eligible. Of these, 31 publications fulfilled the inclusion criteria (Figure 2). 12 studies were considered with fair quality, and 19 with good quality according to the quality assessment.

\section{1 records identified through} databases search

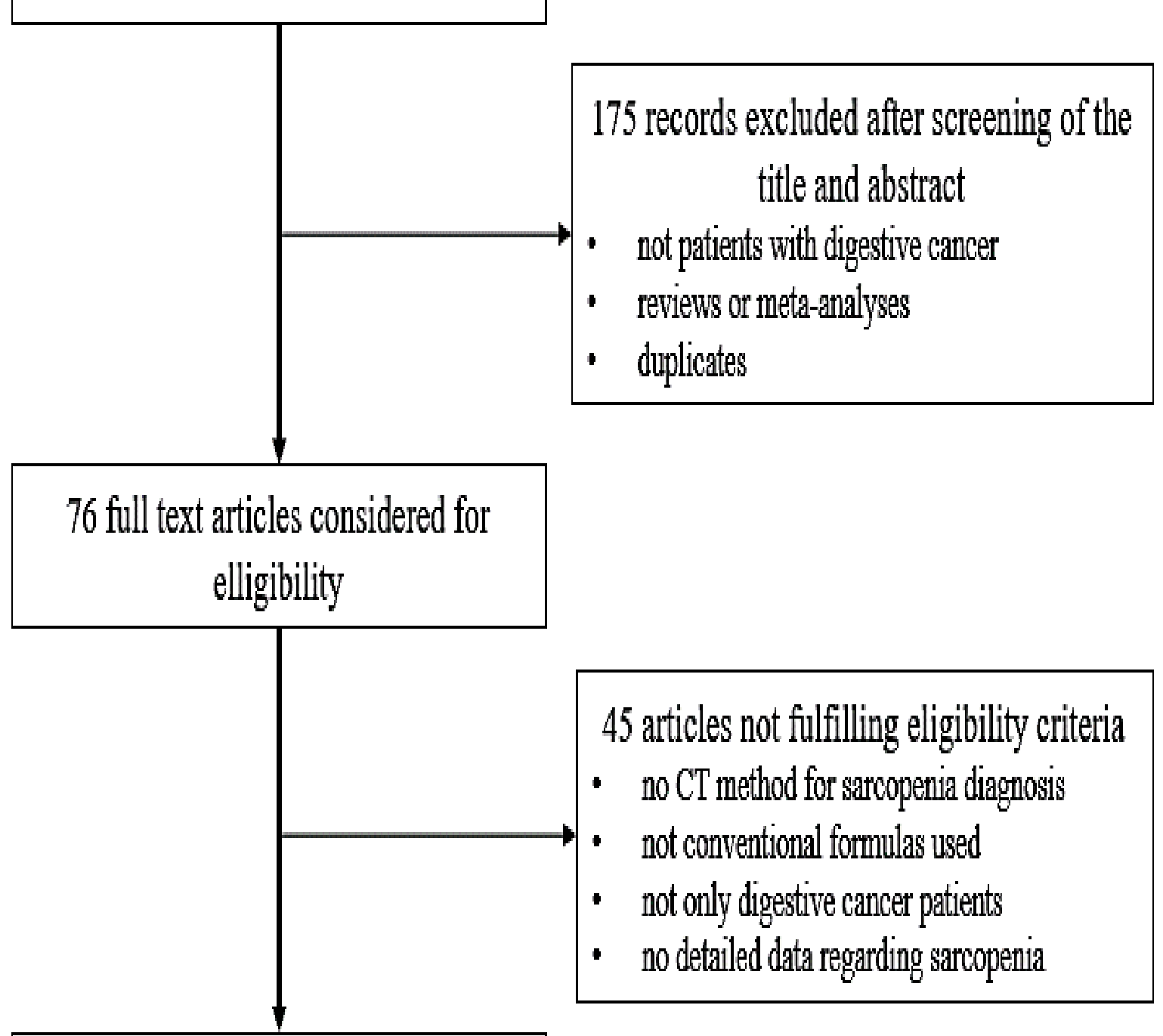

\section{1 articles included in the final} analyses

Figure 2. PRISMA chart. 25 were retrospective cohort studies, and 6 were prospective cohort studies. A summary of the main characteristics of the included studies is provided in Table 1. 


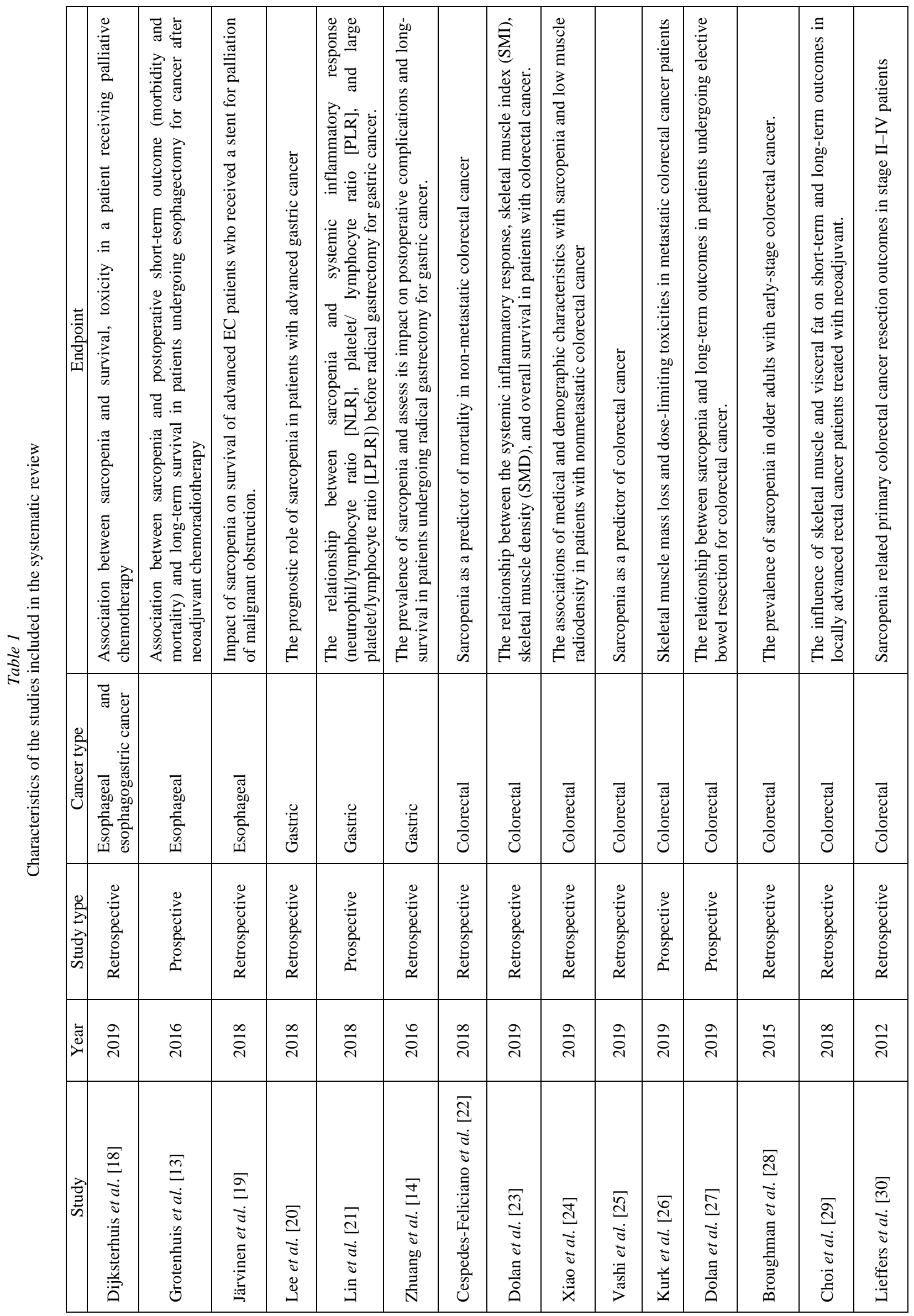




\begin{tabular}{|c|c|c|c|c|c|c|c|c|c|c|c|c|c|c|c|}
\hline 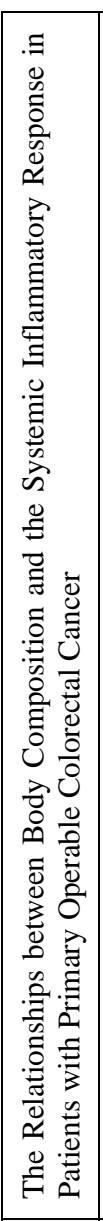 & 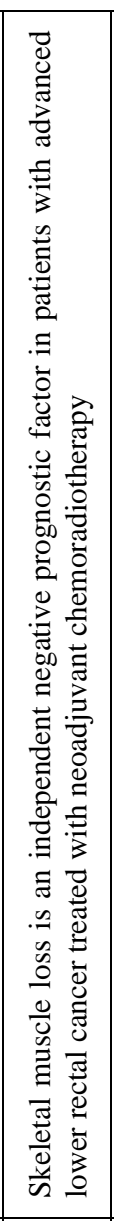 & 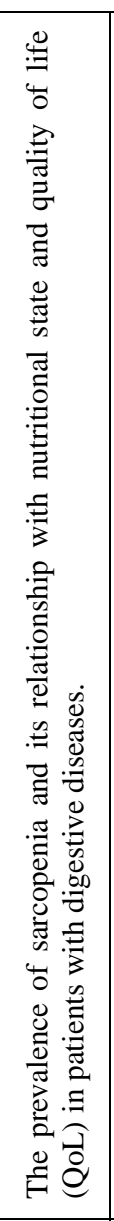 & 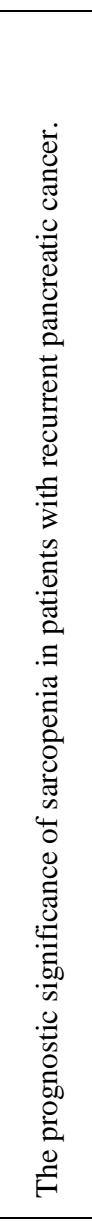 & 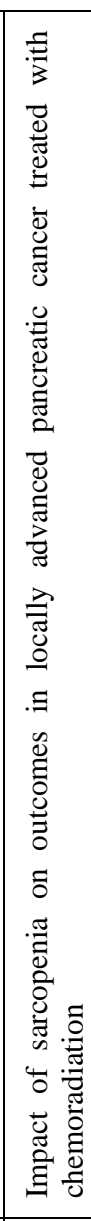 & 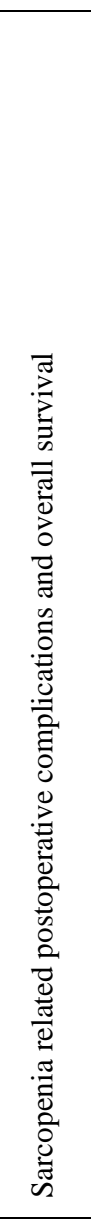 & 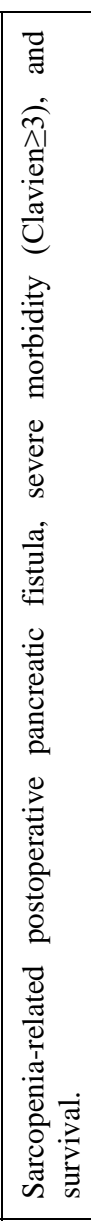 & 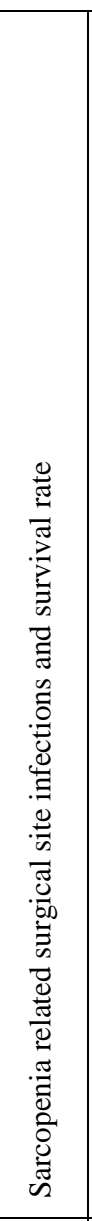 & 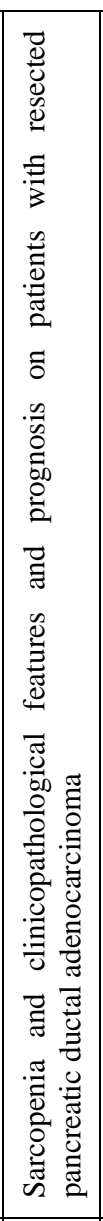 & 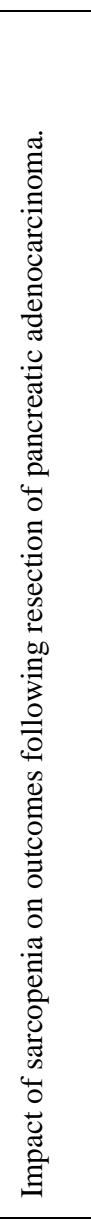 & 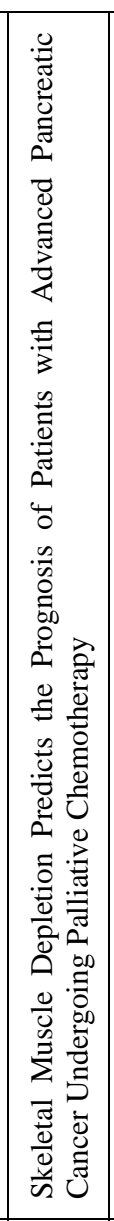 & 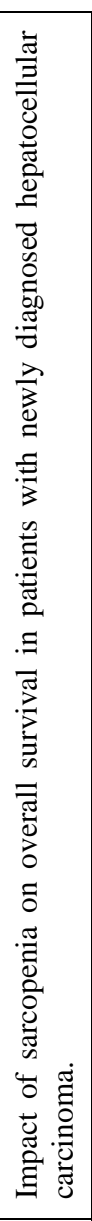 & 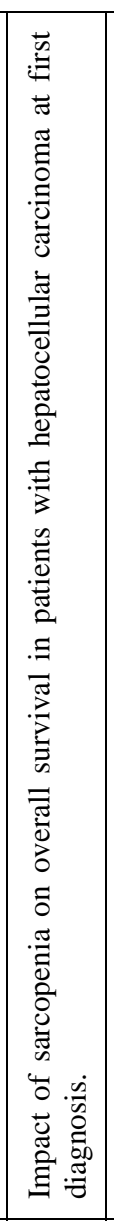 & 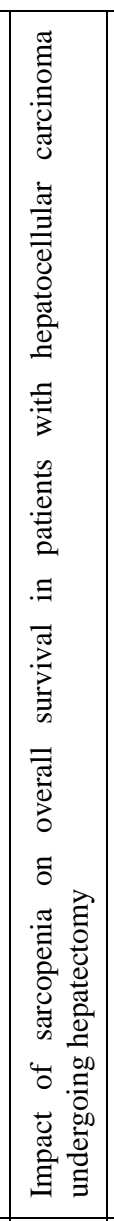 & 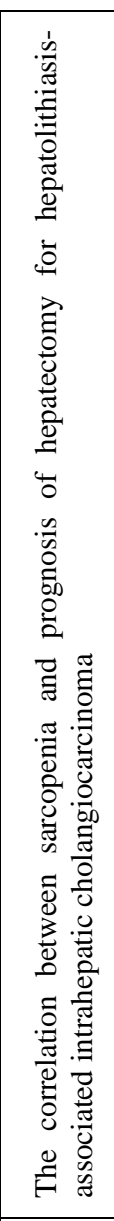 & 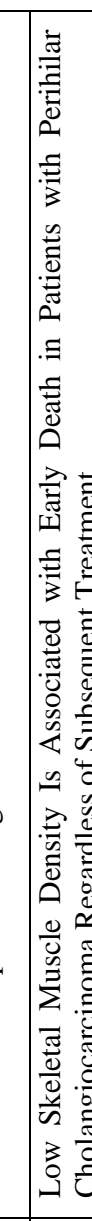 \\
\hline 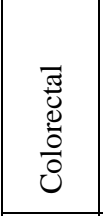 & 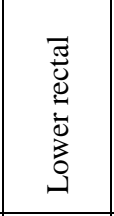 & 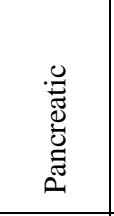 & 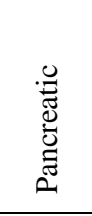 & 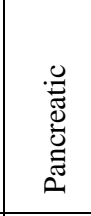 & 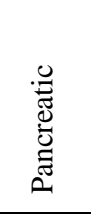 & 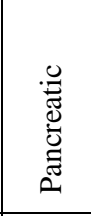 & 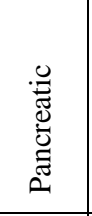 & 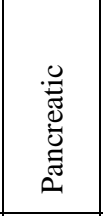 & 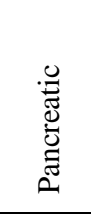 & 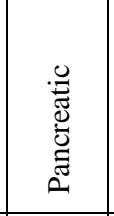 & $\begin{array}{l}\text { 总 } \\
\text { 竞 }\end{array}$ & 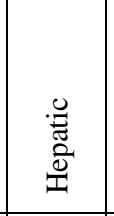 & $\begin{array}{l}\text { 兹 } \\
\text { 畜 }\end{array}$ & 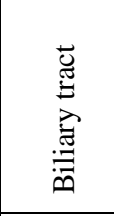 & 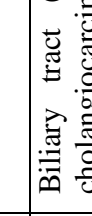 \\
\hline 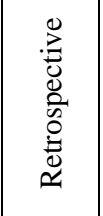 & 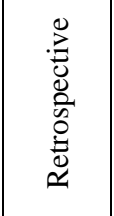 & 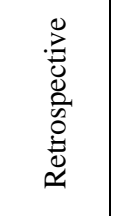 & 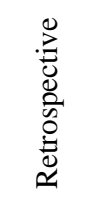 & 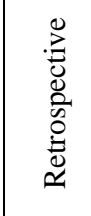 & 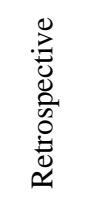 & 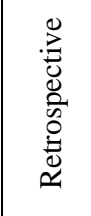 & 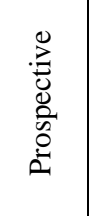 & 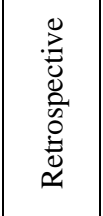 & 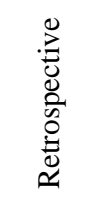 & 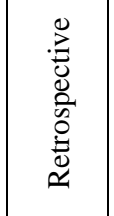 & 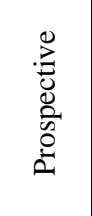 & 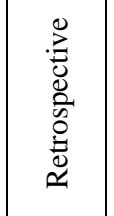 & 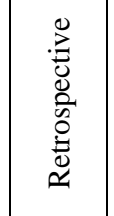 & 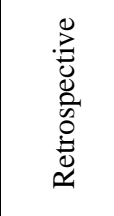 & \\
\hline స̃ & $\stackrel{\infty}{\stackrel{2}{0}}$ & 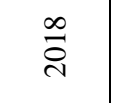 & రి & 亏े & $\stackrel{े}{\vec{i}}$ & $\stackrel{\infty}{\stackrel{2}{\pi}}$ & $\stackrel{\vec{i}}{\vec{i}}$ & $\overrightarrow{\tilde{i}}$ & $\stackrel{\sim}{\tilde{N}}$ & $\stackrel{n}{a}$ & $\stackrel{\infty}{\stackrel{1}{\pi}}$ & $\stackrel{\bar{a}}{0}$ & ŏ & $\stackrel{n}{a}$ & \\
\hline 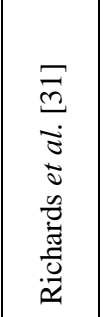 & 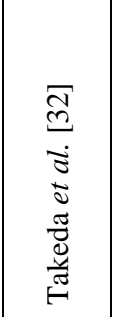 & 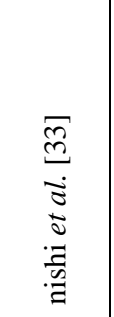 & 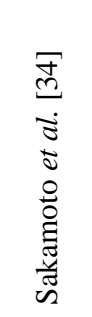 & 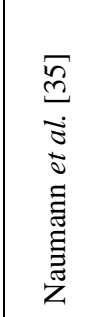 & 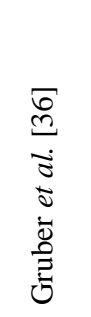 & 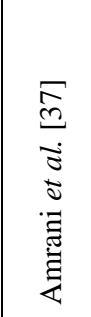 & 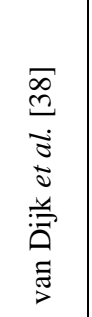 & 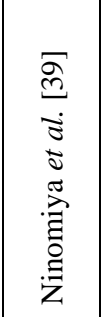 & $\frac{\bar{\partial}}{\stackrel{\partial}{5}}$ & 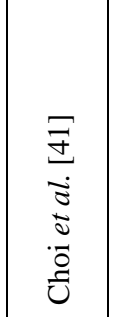 & 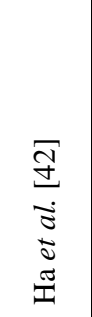 & 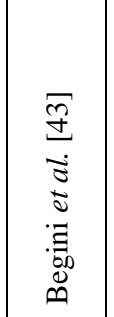 & 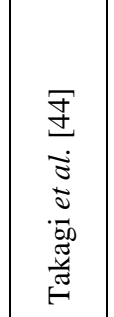 & 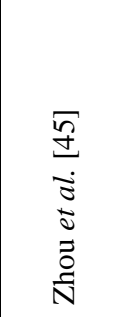 & 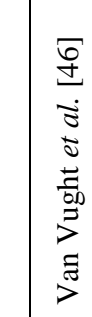 \\
\hline
\end{tabular}




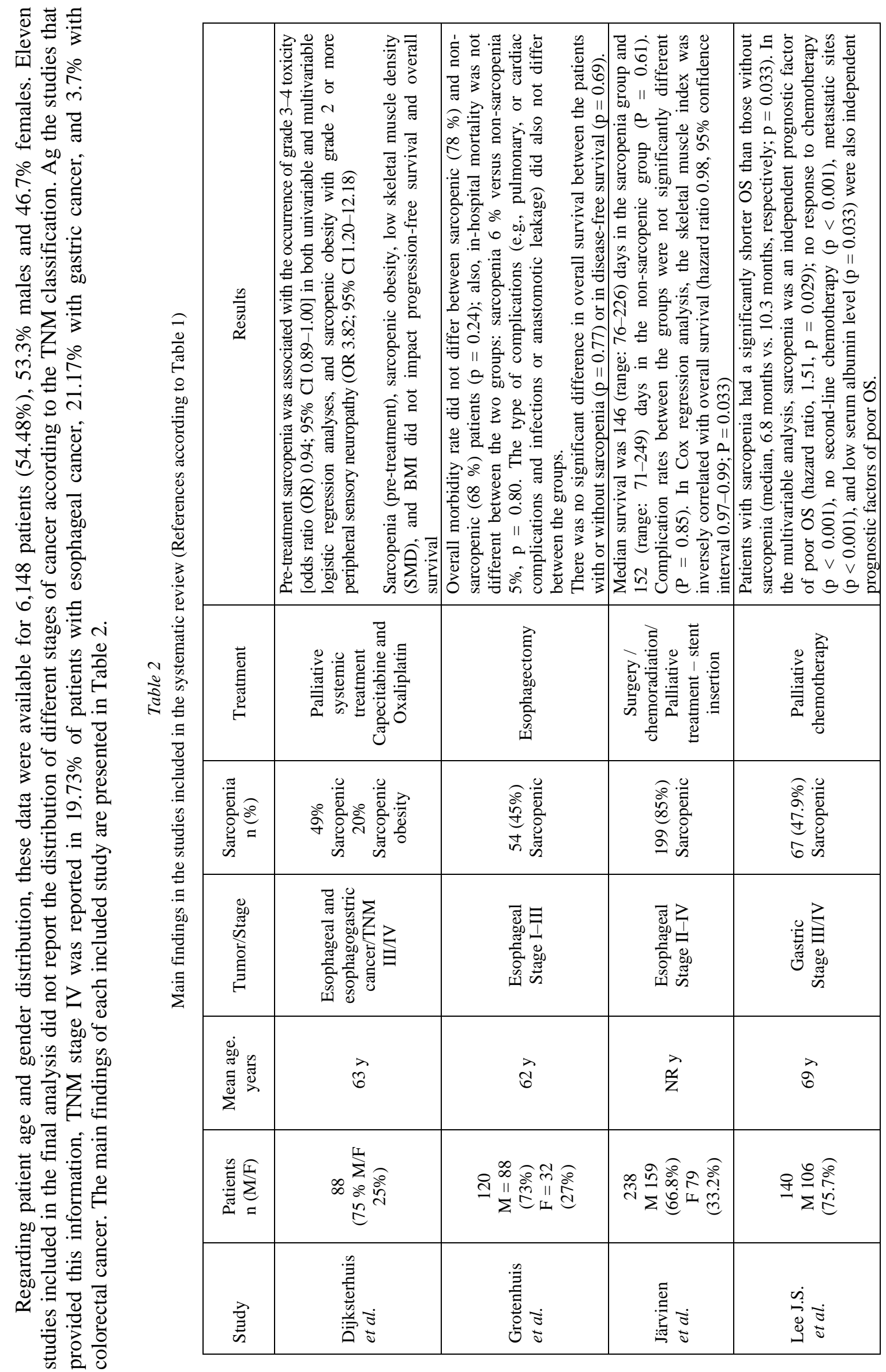




\begin{tabular}{|c|c|c|c|c|c|c|}
\hline 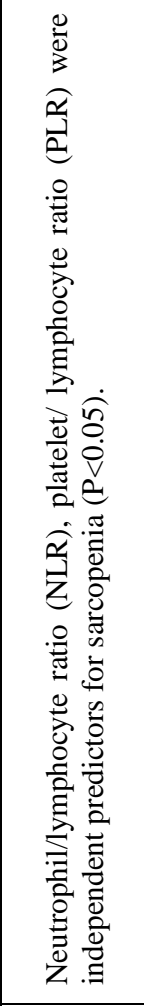 & 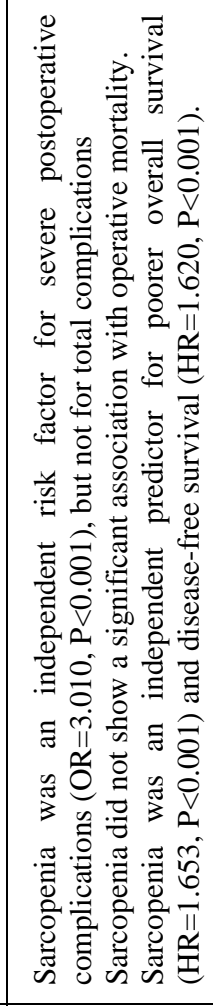 & 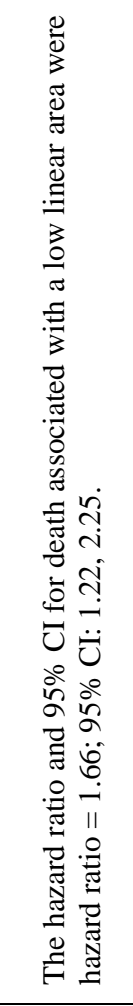 & 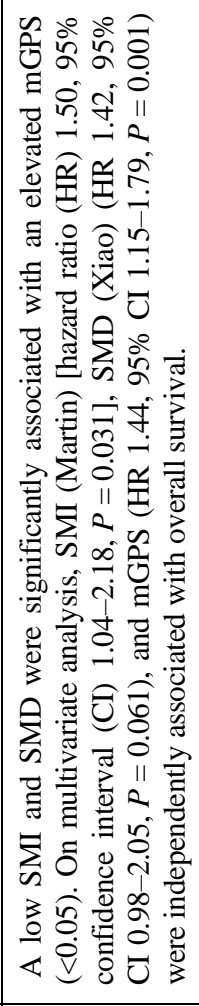 & 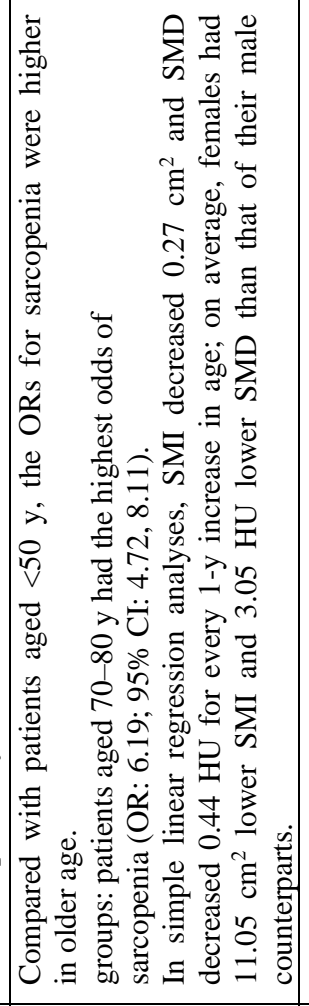 & 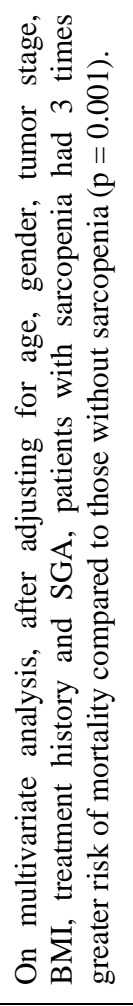 & 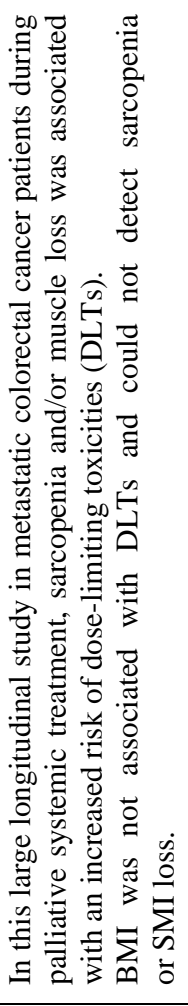 \\
\hline 学 & 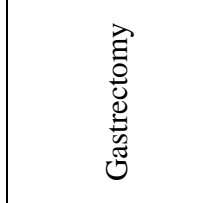 & 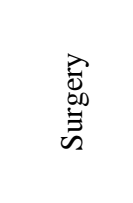 & $\begin{array}{l}\stackrel{\vec{D}}{0} \\
\vdots \\
\vdots \\
\vdots\end{array}$ & $\begin{array}{l}\vec{D}_{0} \\
\vdots \\
\vdots \\
\bar{n}\end{array}$ & 吕 & 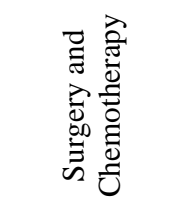 \\
\hline 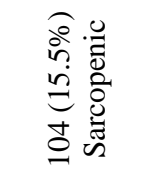 & 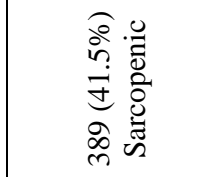 & 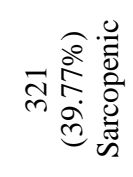 & 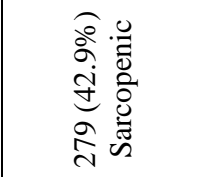 & 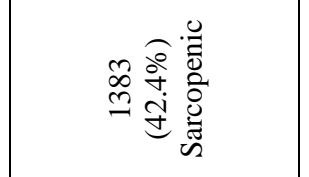 & 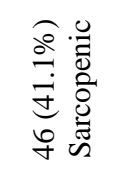 & 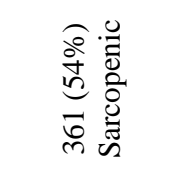 \\
\hline 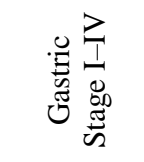 & 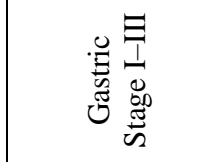 & 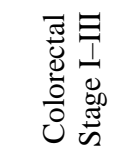 & 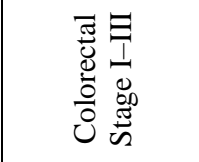 & 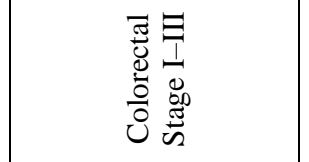 & $\begin{array}{l}\frac{\bar{J}}{0} \\
\frac{0}{0} \\
0 \\
0\end{array}$ & 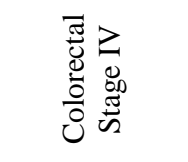 \\
\hline 号 & d & $\frac{2}{6}$ & 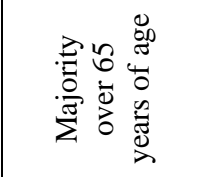 & 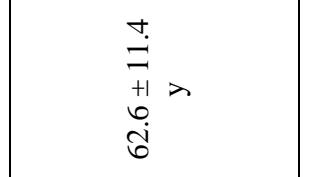 & $\vec{m}$ & ते \\
\hline 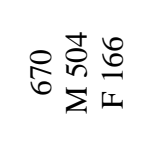 & 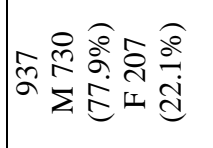 & 客宗客 & 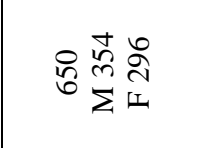 & 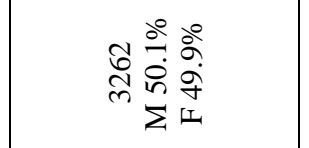 & $\stackrel{0}{z}^{\frac{n}{z}}$ & 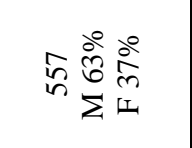 \\
\hline$\vec{\Xi} \Xi$ & 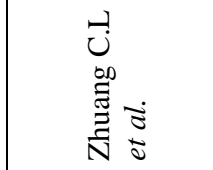 & 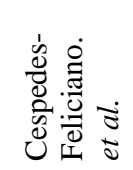 & 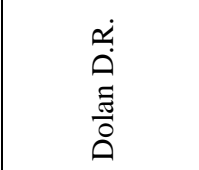 & 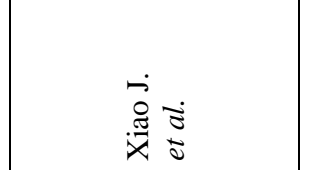 & 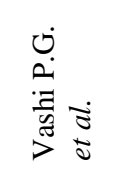 & 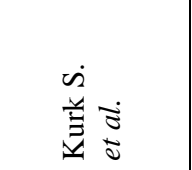 \\
\hline
\end{tabular}




\begin{tabular}{|c|c|c|c|c|c|c|}
\hline 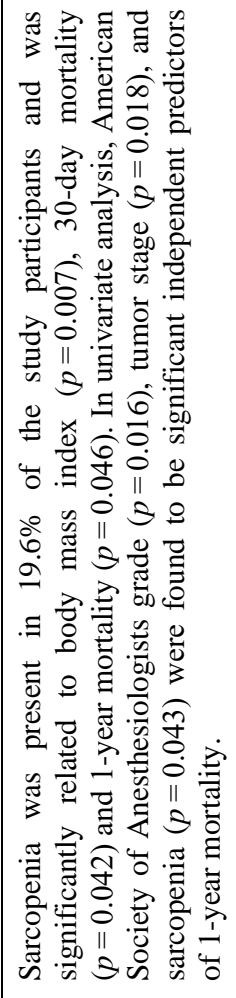 & 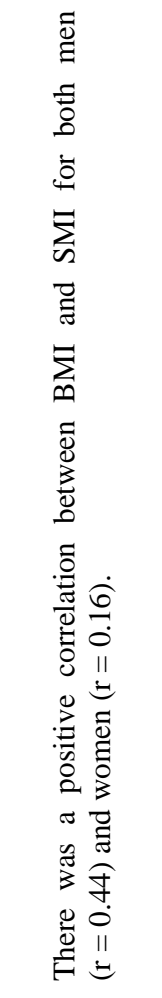 & 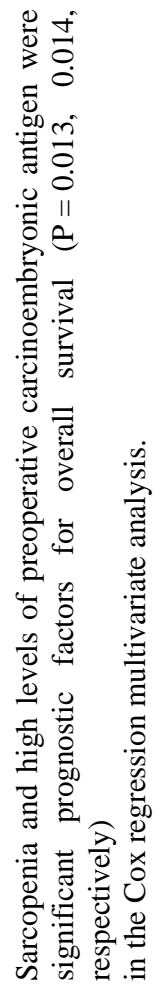 & 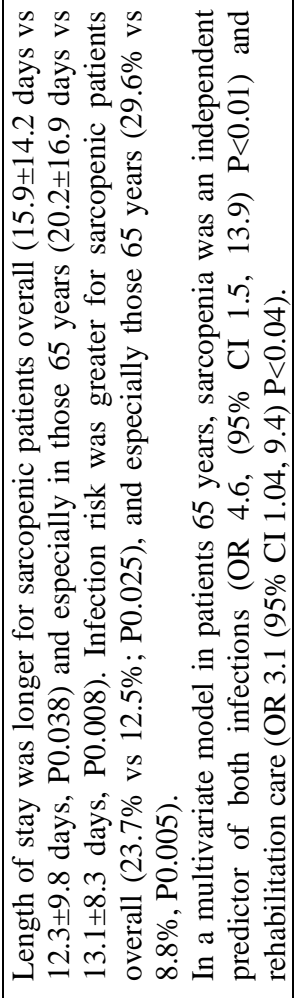 & 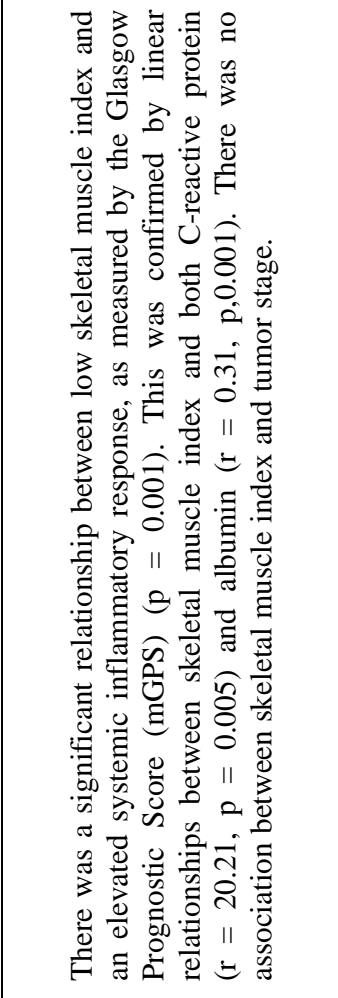 & 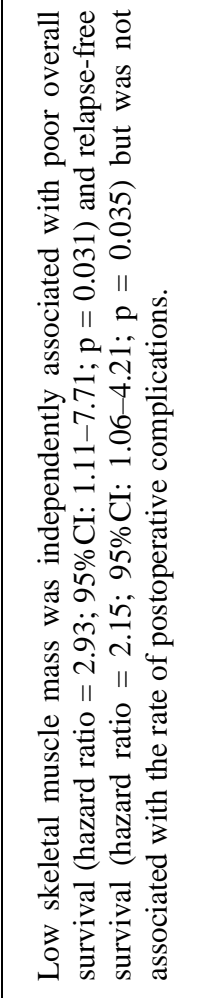 & 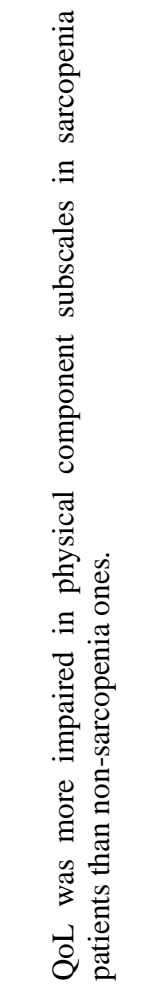 \\
\hline $\begin{array}{l}\vec{D} \\
\stackrel{0}{0} \\
\vdots \\
\bar{\omega}\end{array}$ & 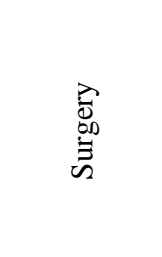 & 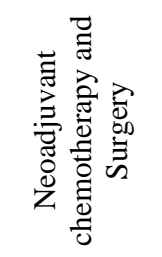 & 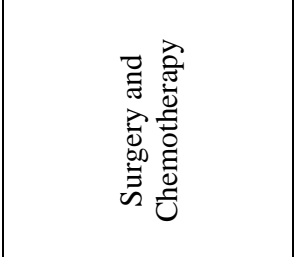 & 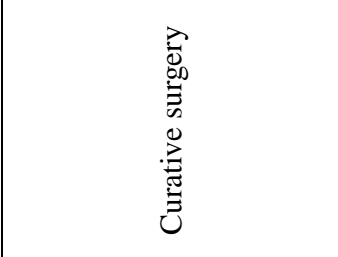 & 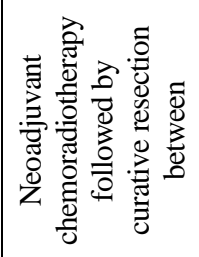 & 总 \\
\hline 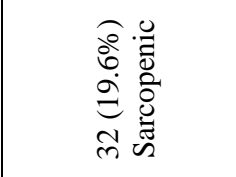 & 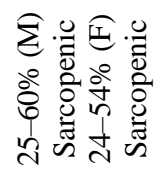 & 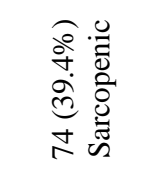 & 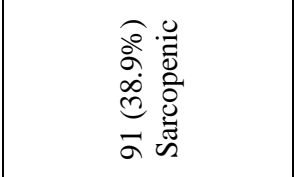 & 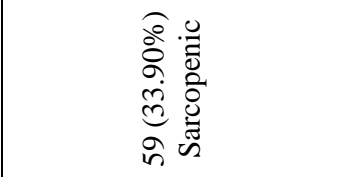 & $\begin{array}{l}\widehat{c} \\
\stackrel{a}{a} \\
a \\
\text { a }\end{array}$ & 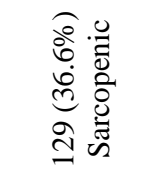 \\
\hline 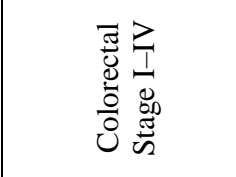 & 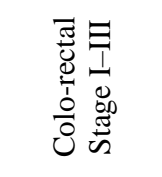 & $\begin{array}{l}\frac{\pi}{0} \\
\frac{0}{5} \\
\frac{0}{0} \\
0\end{array}$ & 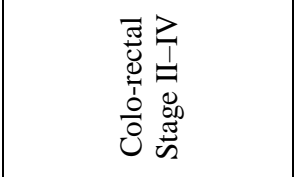 & 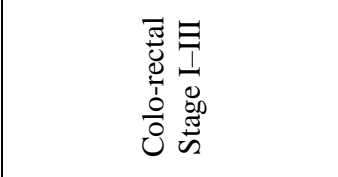 & 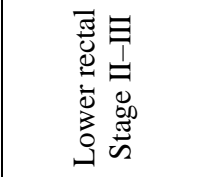 & 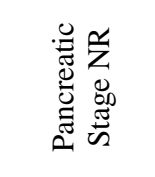 \\
\hline 岂 & $\stackrel{i}{i}$ & 岂 & 号 & 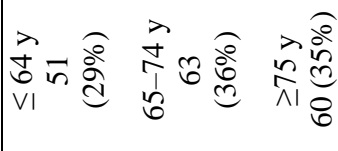 & 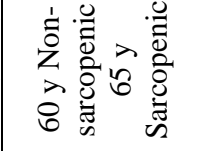 & $\vec{\imath}$ \\
\hline 36 & $\infty \stackrel{\mathcal{F}}{\Sigma} \underset{\Sigma}{\leftarrow}$ & $\stackrel{\infty}{\stackrel{\infty}{-1}}$ & $\stackrel{\overbrace{}}{\sim}$ & 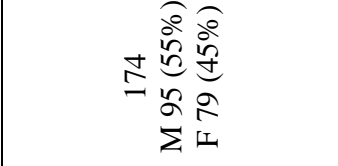 & 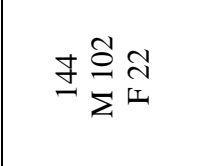 & 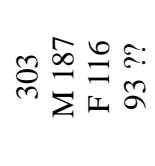 \\
\hline 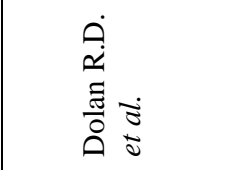 & 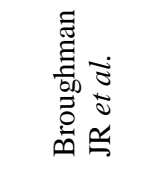 & 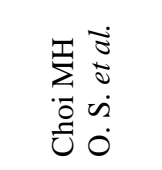 & 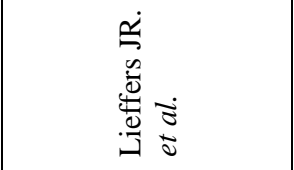 & 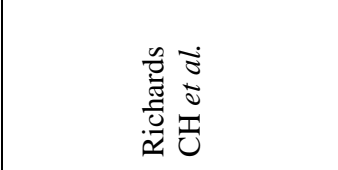 & 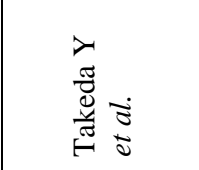 & 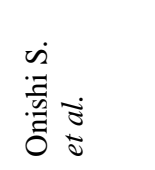 \\
\hline
\end{tabular}




\begin{tabular}{|c|c|c|c|c|c|}
\hline 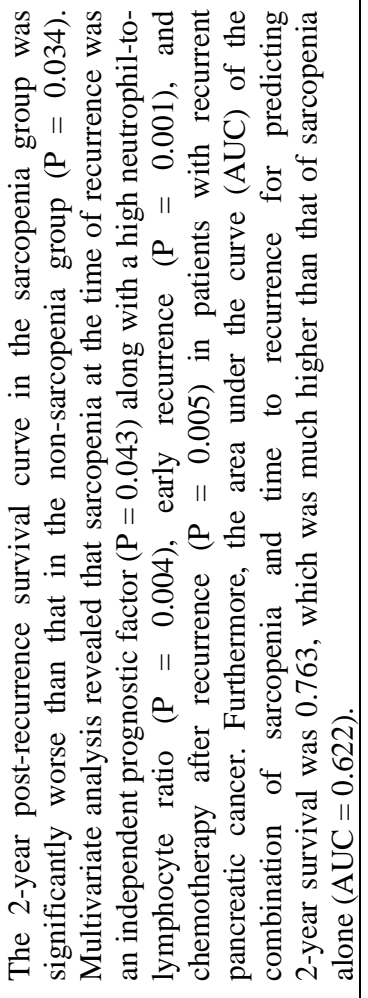 & 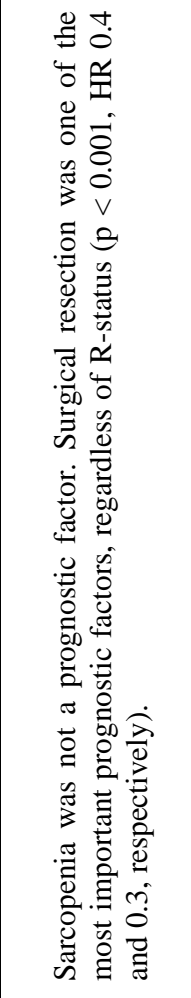 & 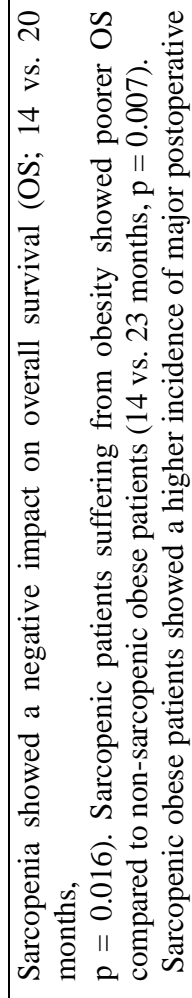 & 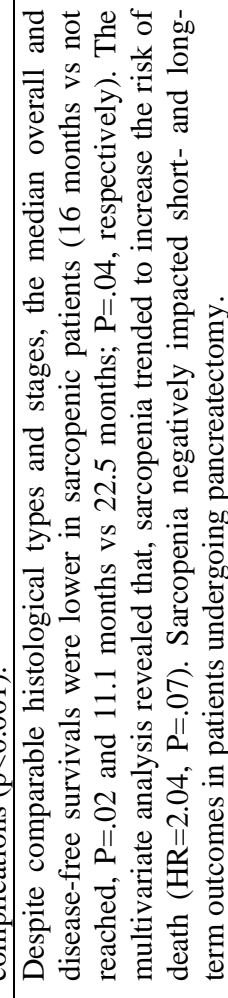 & 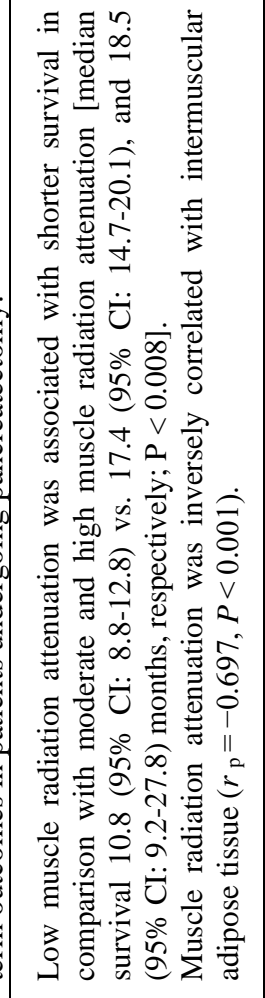 & 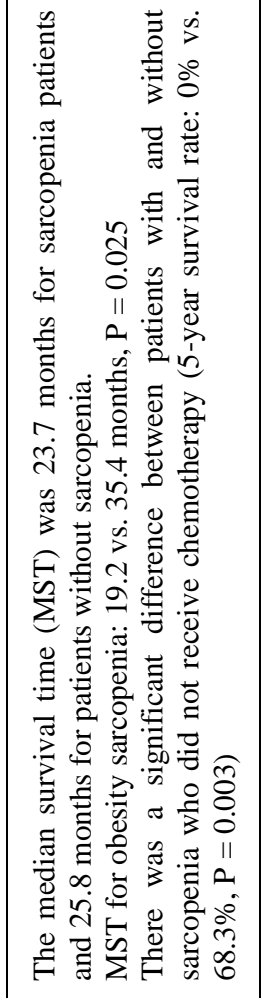 \\
\hline 号 & 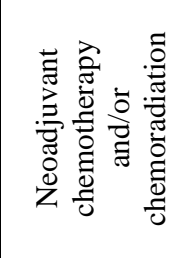 & 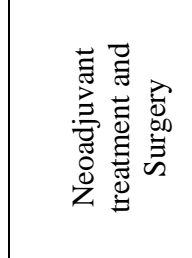 & 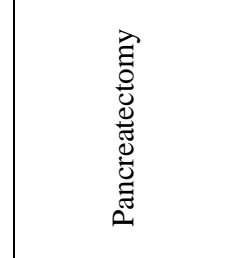 & 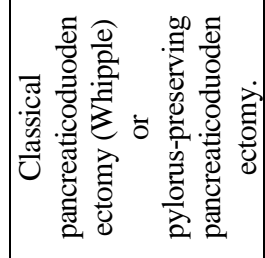 & 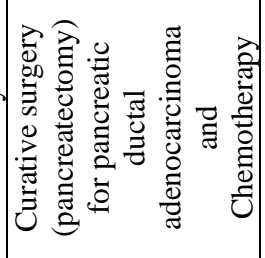 \\
\hline 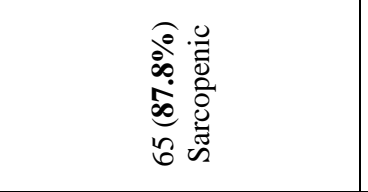 & 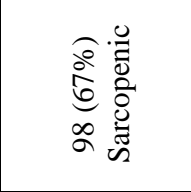 & $\begin{array}{l}\widehat{D} \\
b \\
\infty \\
\infty \\
\infty \\
\infty\end{array}$ & $\begin{array}{l}\frac{0}{2} \\
\text { 巳 } \\
\text { in }\end{array}$ & 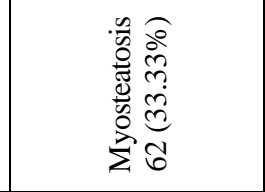 & 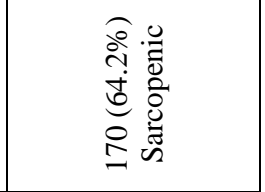 \\
\hline 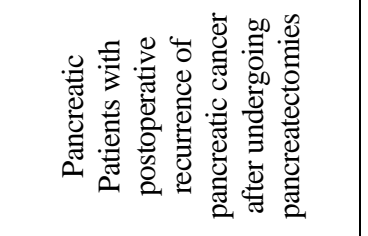 & 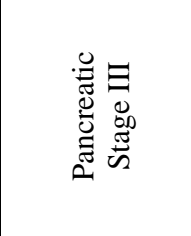 & 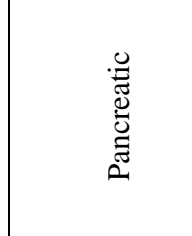 & 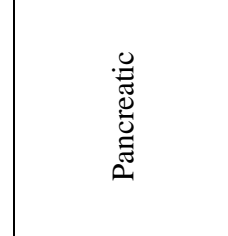 & 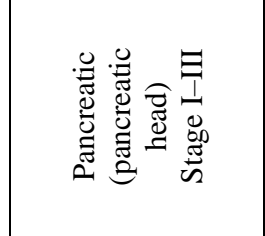 & 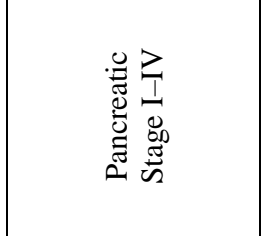 \\
\hline 岂 & $\begin{array}{l}\vec{b} \\
\overrightarrow{0}\end{array}$ & 占 & $\overline{6}$ & $\begin{array}{l}2 \\
n \\
0 \\
0\end{array}$ & $\vec{n}$ \\
\hline$\stackrel{\searrow}{\sim}$ & 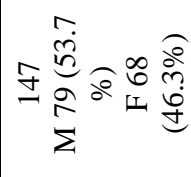 & 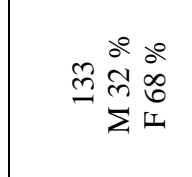 & 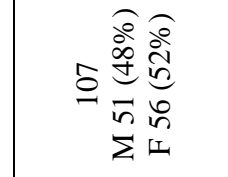 & 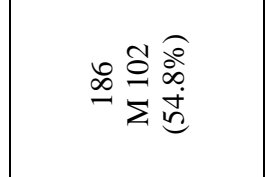 & 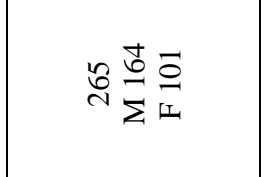 \\
\hline 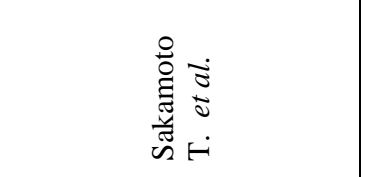 & 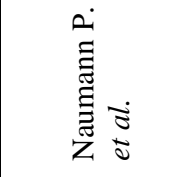 & 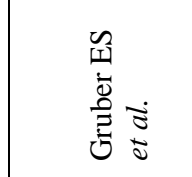 & 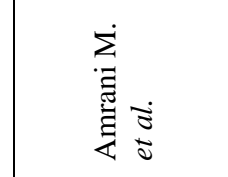 & 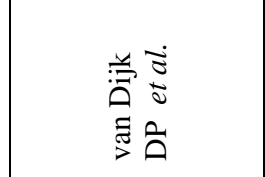 & 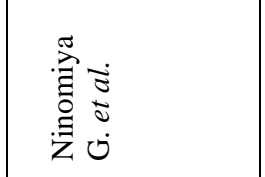 \\
\hline
\end{tabular}




\begin{tabular}{|c|c|c|c|c|}
\hline 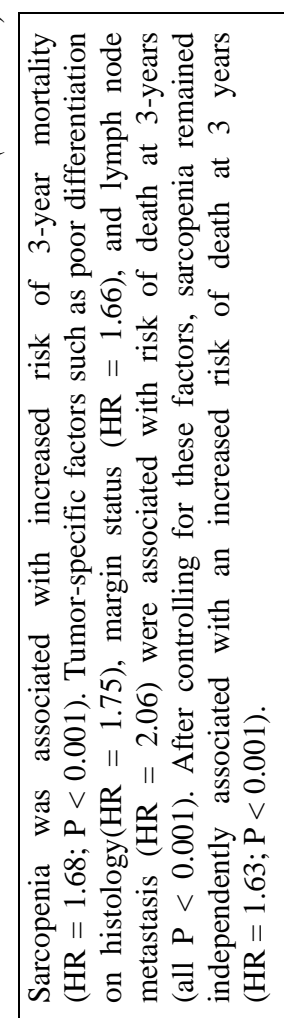 & 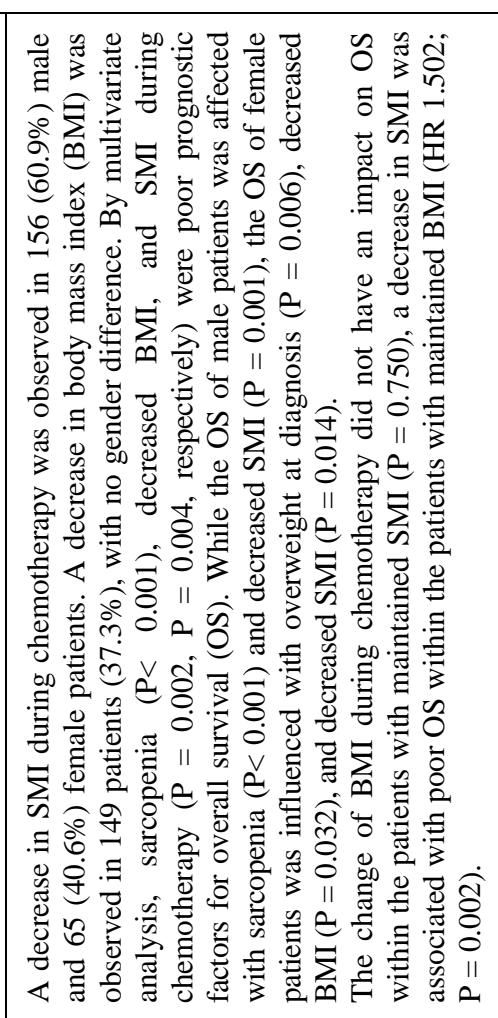 & 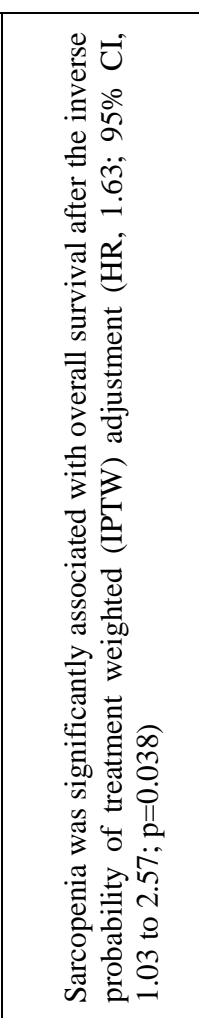 & 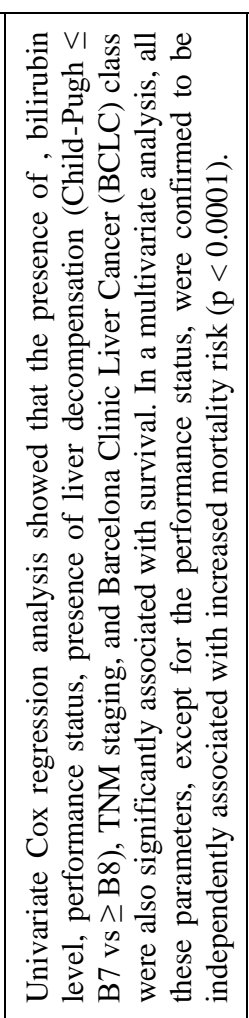 & 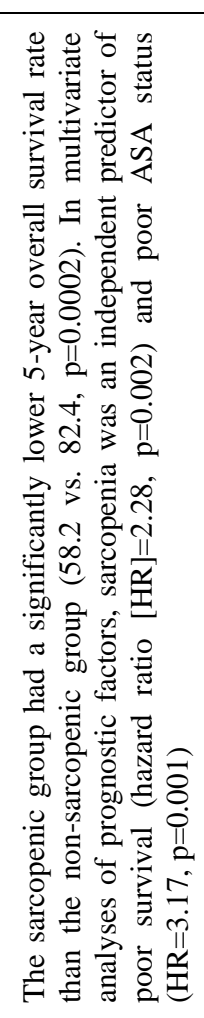 \\
\hline 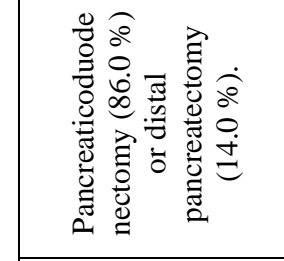 & 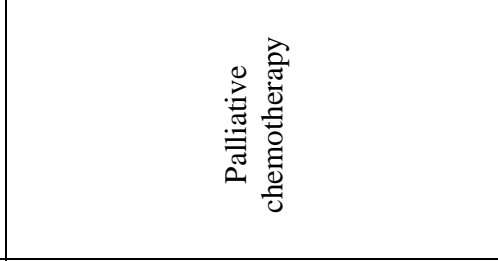 & 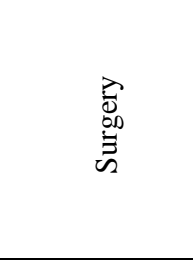 & 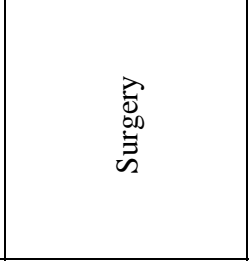 & 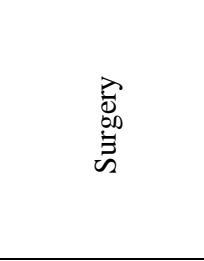 \\
\hline & 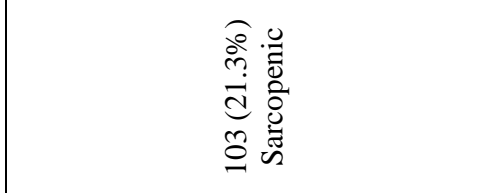 & 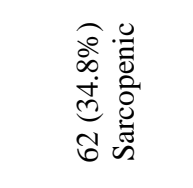 & 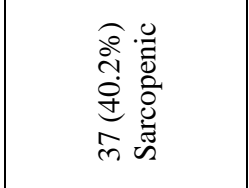 & 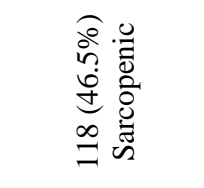 \\
\hline 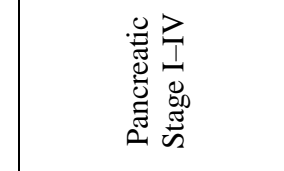 & 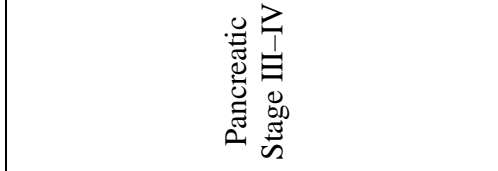 & $\begin{array}{l}\text { 遌 } \\
\text { 竧 }\end{array}$ & $\begin{array}{l}\text { 誉 } \\
\text { 产 }\end{array}$ & $\begin{array}{l}\text { 善 } \\
\text { 厗 }\end{array}$ \\
\hline 离 & 索 & तें & $\frac{\overrightarrow{2}}{\hat{r}}$ & ते \\
\hline 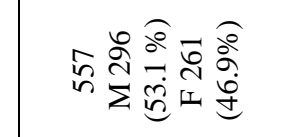 & 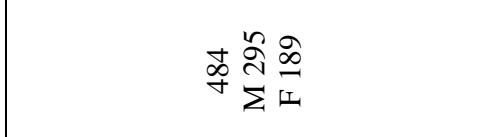 & 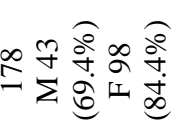 & 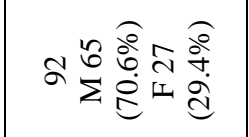 & 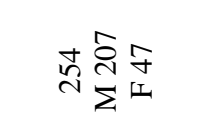 \\
\hline 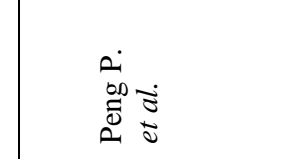 & 氖 & 표 & ๓ & \\
\hline
\end{tabular}



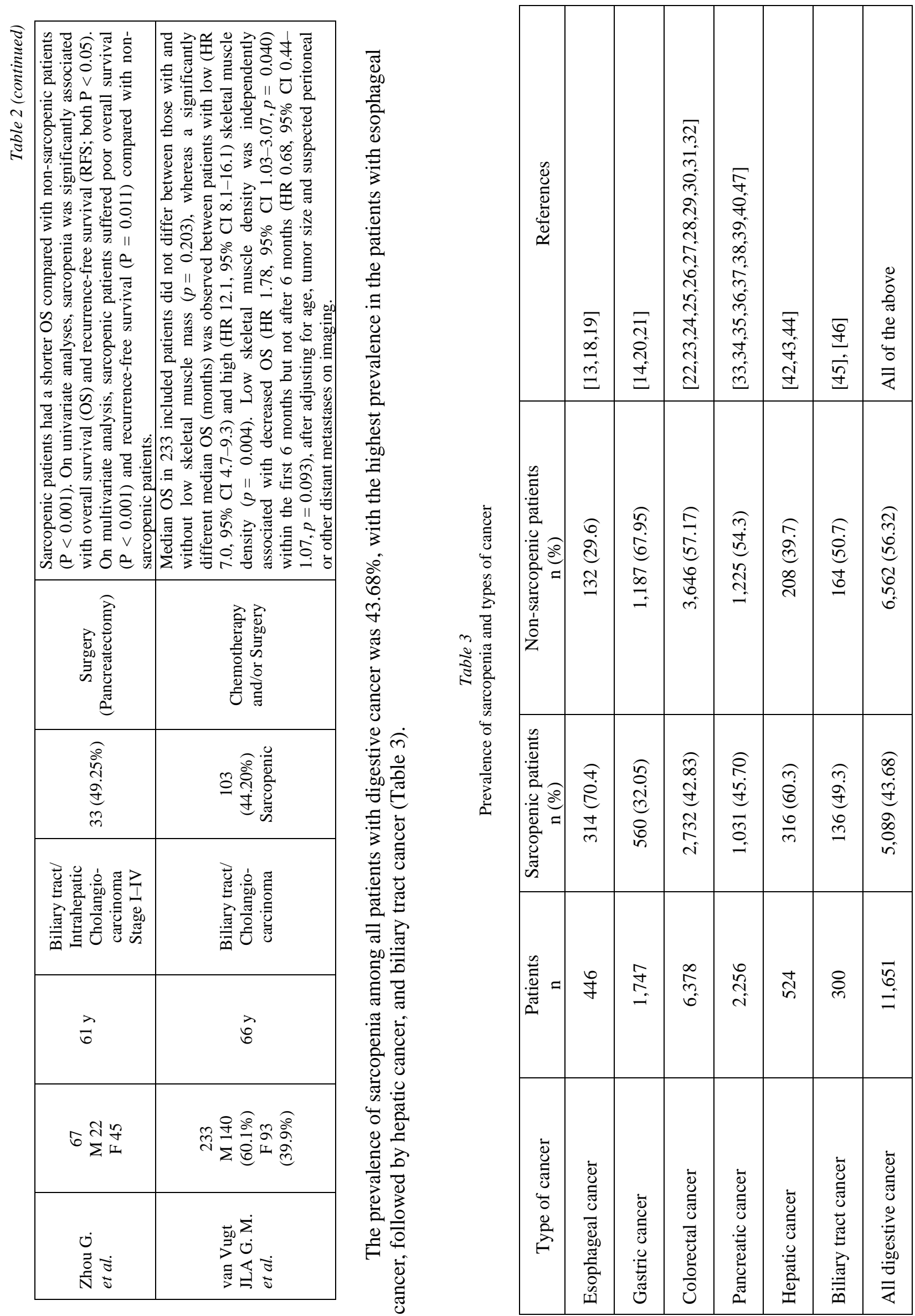
Most patients (93.8\%) underwent curative surgery or surgery and adjuvant chemotherapy. From studies that reported data on gender distribution, the curative treatment was seen slightly more frequently in males (53.3\%).

Only sparse studies reported the prevalence of malnutrition in association with sarcopenia. One study reported the prevalence of sarcopenia in malnourished and wellnourished patients with colorectal cancer. In these patients, sarcopenia was found more often in the malnourished patients compared to wellnourished patients $(55.8 \%$ vs $31.9 \%$, respecttively) [25].

Two studies found a postoperative complications risk higher for sarcopenic patients than for non-sarcopenic patients $[14,30]$, whereas three studies did not find a significant difference in this regard between sarcopenic and non-sarcopenic patients $[19,40,48]$.

Despite the heterogeneity of reported data regarding the mortality, nine studies with digestive cancers, with stages ranging from I to III showed that overall survival and disease-free survival were lower in sarcopenic patients, follow up from 1 year to 5 years postoperatively (Table 4) [23], [26,27,29,37-39,42-46]. Interestingly, one study showed that the median survival time in patients with pancreatic cancer was lower for sarcopenic obesity but not for sarcopenic without obesity [39].

Recurrence rates after operated pancreatic cancer were significantly higher in sarcopenic patients [34].

Table 4

Mortality and sarcopenia in patients with digestive cancer

\begin{tabular}{|c|c|c|c|c|c|c|}
\hline \multirow[t]{2}{*}{ Study } & \multicolumn{2}{|c|}{ Disease-free survival } & \multicolumn{2}{|c|}{ Median survival time } & \multicolumn{2}{|c|}{ Mortality } \\
\hline & Sarcopenia & $\begin{array}{c}\text { Non- } \\
\text { sarcopenia }\end{array}$ & Sarcopenia & $\begin{array}{c}\text { Non- } \\
\text { sarcopenia }\end{array}$ & Sarcopenia & $\begin{array}{c}\text { Non- } \\
\text { sarcopenia }\end{array}$ \\
\hline Grotenhuis et al. [13] & $45 \%$ & $55 \%$ & & & $6 \%$ & $4 \%$ \\
\hline $\begin{array}{l}\text { Dijksterhuis WPM } \\
{[18]}\end{array}$ & No: 54 & No: 66 & & & $18 \%$ & $4 \%$ \\
\hline Dolan et al. [23] & $\begin{array}{c}36.61 \% \text { at } 5 \\
\text { years }\end{array}$ & $\begin{array}{c}49.53 \% \text { at } 5 \\
\text { years }\end{array}$ & & & & \\
\hline Choi et al. [29] & & & & & $\begin{array}{c}21.6 \% \text { at } 5 \\
\text { years }\end{array}$ & $\begin{array}{c}7.8 \% \text { at } 5 \\
\text { years }\end{array}$ \\
\hline El Amrani et al. [37] & 11.1 months & 22.5 months & & & & \\
\hline van Dijk et al. [38] & 10.8 months & 18.5 months & & & & \\
\hline Ninomiya et al. [39] & & & 23.7 months & 25.8 months & & \\
\hline Begini et al. [43] & & & 66 weeks & 123 weeks & & \\
\hline Takagi et al. [44] & $58.2 \%$ & $82.4 \%$ & & & & \\
\hline
\end{tabular}

The rest of the studies expressed the relationship between sarcopenia and mortality through multivariate analyzes, CI and HR or had other endpoints, such as: the relationship between sarcopenia and chemotherapy toxicity, the relationship between sarcopenia and systemic inflammatory response and the relationship between sarcopenia and postoperative complications.

\section{DISCUSSIONS}

In our systematic review, we demonstrated a high prevalence of secondary sarcopenia $(43.68 \%)$ in patients with any form of gastrointestinal cancer. A strength of our study resides in the selection of studies with prespecified cut-off points for defining SeS, based on EWGSOP recommendations. This approach may provide a prevalence closer to reality compared to analysis including studies with cut-off points relying on the data within.

Our analysis showed $\mathrm{SeS}$ is not uniformly distributed among different types of digestive cancer. The highest incidence was seen in esophageal cancer which may be explained by the fact this type of cancer is usually accompanied by difficulties of feeding. The lowest prevalence of $\mathrm{SeS}$ was seen in patients with gastric cancer. We were not able to perform a reliable analysis of the prevalence of $\mathrm{SeS}$ based on different TNM stages 
because of a lack of reporting necessary data in the included studies. However, the present study signal for anon-uniform distribution may provide a starting point for future risk stratification for $\mathrm{SeS}$ based on the type of cancer and grading.

The relationship between $\mathrm{SeS}$ and postoperative complications remains unclear, only two studies from our selection demonstrating a higher incidence of such complications in sarcopenic patients. However, a recent meta-analysis showed $\mathrm{SeS}$ as a predictor for postoperative complications in patients with gastrointestinal neoplasms [49]. Further trials should assess more clearly the impact of sarcopenia on curative outcomes not only concerning the type of cancer but probably also other factors, like TNM staging, to elaborate effective pre-operative management of these patients.

Nine studies demonstrated increased mortality in patients with digestive cancer and SeS. In one study the mortality was up to 3 times higher in sarcopenic patients [25]. The assessment of the role of $\mathrm{SeS}$ on the survival rate of these patients is difficult, as there are different factors, more or less known, that may contribute to the prognosis. For example, in the case of esophageal cancer, it is characteristic to associate eating disorder that may lead to malnutrition and cachexia, making difficult to analyze the direct impact of sarcopenia in the evolution of such patients. Among patients with esophageal cancer, stage IV (TNM), toxicity-related chemotherapy, and survival were not associated with sarcopenia [18]. Also, in stage III TNM esophageal cancer treated with neoadjuvant chemoradiotherapy followed by esophagectomy, the presence of sarcopenia was not associated with negative shortand long-term outcome [13,50]. Conversely, one study showed that patients with gastric cancer who had preoperative sarcopenia and were converted to non-sarcopenic status did not present major postoperative complications [51]. Also, the phenotype of sarcopenia may play a role. In patients with pancreatic cancer, the sarcopenia associated with obesity had lower survival time compared to non-obese sarcopenia [39].

Given the impact of sarcopenia, the accuracy of diagnosis is of utmost importance. We included in our analysis only studies that diagnosed sarcopenia based on the CT scan method. This is a reliable method regardless of the software (like FatSeg, OsiriX, ImageJ, and sliceOmatic) used for measurements [52]. Various calculation formulas for sarcopenia are found in the literature. For obtaining comparable results it would be ideal to use a unique calculation formula. Therefore, we included in our study only trials that used the most recommended formulas for the diagnosis of SeS, meaning SMI based on skeletal muscle mass or skeletal muscle area parameters [1,4]. However, the CT method is difficult and time-consuming, requiring specialized equipment and personnel, thus not always available. Further research for a new less timeconsuming and cheaper diagnostic tool for $\mathrm{SeS}$, simpler and reproducible, is necessary.

Lastly, the early diagnosis of sarcopenia and timing of therapeutic intervention may reflect on costs, as the hospitalization costs are significantly higher in sarcopenic patients [47].

\section{LIMITATIONS}

Our review has several limitations. The distribution of the number of patients among types of digestive cancers is significantly different. Many studies did not provide data related to cancer staging and the impact of sarcopenia in surgical and chemo/radiotherapy treatment. Thus, the data regarding the relationship between sarcopenia and curative treatments or severity of the disease is limited.

There is a risk of bias regarding the diagnosis of sarcopenia. Even if the diagnosis of sarcopenia was made using the CT scan method at the level of the lumbar vertebra 3, the SMI was calculated using different formulas. Also, the prevalence of SeS may differ if diagnostic methods other than CT are used.

\section{CONCLUSIONS}

1) Secondary sarcopenia is highly prevalent among patients with digestive cancer.

2) It was most frequently associated with esophageal cancer, followed by hepatic and biliary tract cancer. The least prevalence was in gastric cancer.

3) It seems the presence of sarcopenia has a negative impact on outcomes of curative treatments and survival rate, but a definite role is unclear.

4) For clarifying this issue, a uniform method of diagnosis and detailed data regarding staging and outcomes in patients with digestive cancer and sarcopenia are needed from future studies. 
Introducere. Sarcopenia se caracterizează printr-o scădere a masei musculare scheletice, asociată cu forță musculară scăzută și/sau performanță fizică slabă. Evaluarea prevalenței sarcopeniei printre cancerele digestive și stabilirea impactului pe care sarcopenia îl are asupra evoluției postoperatorii a tumorilor digestive pot reprezenta un pilon central în îmbunătățirea rezultatelor postoperatorii prin îngrijirea sarcopeniei perioperatorii.

Această scurtă trecere în revistă a avut drept scop evaluarea prevalenței sarcopeniei la pacienții cu cancer digestiv.

Materiale și metodă. Baza de date PubMed a fost căutată pentru „, sarcopenie” și „,cancere digestive” de la 1 ianuarie 2010, până la 30 septembrie 2020. Ghidul PRISMA a fost utilizat pentru această revizuire sistematică. După procesul de selecție, 31 de studii complete au fost incluse in analiza noastră.

Evaluarea diagnosticului de sarcopenie pentru studiile incluse în această revizuire sistematică $s$-a bazat pe un calcul tomografic computerizat al indicelui mușchilor scheletici la a treia vertebră lombară.

Rezultate. Dintr-un un total de 11651 pacienți cu cancer digestiv, prevalența sarcopeniei a fost de 43,68\%.

Cea mai mare prevalență a pacienților sarcopenici a fost în cancerul esofagian $(70,4 \%)$ și hepatic (60,3\%), urmat de tractul biliar (49,3\%), pancreatic $(45,70 \%)$, colorectal $(42,83 \%)$ și cancer gastric (32,05\%) cu cea mai mică prevalență.

Rezultatele studiilor efectuate până acum cu privire la prevalența sarcopeniei in cancerele digestive și relevanța acesteia în evoluția acestor tipuri de cancer sunt discordante și inegale.

Unele studii arată că prezența sarcopeniei la pacienții cu cancere digestive este asociată cu o rată crescută de complicații postoperatorii, o toxicitate crescută a chimioterapiei și o mortalitate crescută. Alte studii nu găsesc sarcopenia ca factor de risc independent asociat cu consecințe negative la pacienții cu cancere digestive.

Concluzii. Sarcopenia este răspândită în cancerele digestive. Încă nu există un consens cu privire la impactul sarcopeniei asupra tratamentului cancerelor digestive. Sunt necesare studii suplimentare pentru a evalua consecințele reale ale sarcopeniei în cancerele digestive.

Correspondence to: Carmen Haiducu, M.D, Carol Davila University of Medicine and Pharmacy Bucharest, Romania. E-mail: haiducu_carmen@yahoo.com. Phone: 0040769655331

Conflict of interest disclosure: The authors declare there is no conflict of interest.

\section{REFERENCES}

1. CRUZ-JENTOFT, A.J., BAHAT, G., BAUER, J., BOIRIE, Y., BRUYÈRE, O., CEDERHOLM, T., et al. Sarcopenia: Revised European consensus on definition and diagnosis. Age Ageing 2019;48(1):16-31.

2. VON HAEHLING, S., MORLEY, J.E., ANKER, S.D.An overview of sarcopenia: Facts and numbers on prevalence and clinical impact. b.n. Available at: doi:10.1007/s13539-010-0014-2.

3. HEYMSFIELD, S.B., WANG, Z.M., BAUMGARTNER, R.N., ROSS, R. Human body composition: Advances in models and methods. b.n.Available at: doi:10.1146/annurev.nutr.17.1.527.

4. PRADO, C.M., LIEFFERS, J.R., MCCARGAR, L.J., REIMAN, T., SAWYER, M.B., MARTIN, L., et al. Prevalence and clinical implications of sarcopenic obesity in patients with solid tumours of the respiratory and gastrointestinal tracts: a population-based study. Lancet Oncol. 2008;9(7).

5. RIER, H.N., JAGER, A., SLEIJFER, S., MAIER, A.B., LEVIN, M. The Prevalence and Prognostic Value of Low Muscle Mass in Cancer Patients: A Review of the Literature. Oncologist 2016;21(11).

6. MALTAIS ML, DESROCHES J, DIONNE IJ. Changes in muscle mass and strength after menopause. J Musculoskelet Neuronal Interact. 2009;9(4):186-97. https://www.ncbi.nlm.nih.gov/pubmed/19949277. no date. 
7. GOODPASTER BH, PARK SW, HARRIS TB, KRITCHEVSKY SB, NEVITT M, SCHWARTZ AV, SIMONSICK EM, TYLAVSKY FA, VISSER M, NEWMAN AB. The loss of skeletal muscle strength, mass, and quality in older adults: the health, aging and body composition study. J Gerontol A B no date.

8. GALLAGHER D, VISSER M, DE MEERSMAN RE, SEPULVEDA D, BAUMGARTNER RN, PIERSON RN, HARRIS T, HEYMSFIELD SB. Appendicular skeletal muscle mass: effects of age, gender, and ethnicity. J Appl Physiol (1985). 1997;83(1):229-39. no date.

9. https://www.visiblebody.com/learn/skeleton/appendicular-skeleton.

10. NIRMALA RATHNAYAKE, GAYANI ALWIS LEKAMWASAM, J.L. AND S. Concordance between appendicular skeletal muscle mass measured with DXA and estimated with mathematical models in middle-aged women. Rathnayake al. J. Physiol. Anthropol. 3719 https//doi.org/10.1186/s40101-018-0179-5 no date.

11. WANG J, THORNTON JC, KOLESNIK S, PIERSON RN JR. Anthropometry in body composition: an overview. Ann N Y Acad Sci. 2000;904:317-26. https://www.ncbi.nlm.nih.gov/pubmed/10865763. no date.

12. ROLlAND, Y., CZERWINSKI, S., VAN KAN, G.A., MORLEY, J.E., CESARI, M., ONDER, G., et al. Sarcopenia: Its assessment, etiology, pathogenesis, consequences and future perspectives. b.n.Available at: doi:10.1007/BF02982704.

13. GROTENHUIS, B.A., SHAPIRO, J., VAN ADRICHEM, S., DE VRIES, M., KOEK, M., WIJNHOVEN, B.P.L., et al. Sarcopenia/Muscle Mass is not a Prognostic Factor for Short- and Long-Term Outcome After Esophagectomy for Cancer. World J. Surg. 2016;40(11).

14. ZHUANG, C. LE, HUANG, D.D., PANG, W.Y., ZHOU, C.J., WANG, S.L., LOU, N., et al. Sarcopenia is an independent predictor of severe postoperative complications and long-term survival after radical gastrectomy for gastric cancer: Analysis from a large-scale cohort. Med. (United States) 2016;95(13).

15. MOHER, D., LIBERATI, A., TETZLAFF, J., ALTMAN, D.G. Preferred reporting items for systematic reviews and metaanalyses: the PRISMA statement. BMJ 2009;339(jul21 1):b2535-b2535.

16. CHEN, L.-K., WOO, J., ASSANTACHAI, P., AUYEUNG, T.-W., CHOU, M.-Y., IIJIMA, K., et al. Asian Working Group for Sarcopenia: 2019 Consensus Update on Sarcopenia Diagnosis and Treatment. J. Am. Med. Dir. Assoc. 2020;21(3):300-307.e2.

17. WElls, G., SHEA, B., O’CONNEll, D., PETERSON, J., WElCH, V., LOSOS, M., et al. The Newcastle-Ottawa Scale (NOQAS) for Assessing the Quality of Non-Randomized Studies in Meta-Analysis. Available at http://www.ohri.ca/programs/clinical_epidemiology/oxford.asp. The Ottawa Hospital.

18. DIJKSTERHUIS, W.P.M., PRUIJT, M.J., VAN DER WOUDE, S.O., KLAASSEN, R., KURK, S.A., VAN OIJEN, M.G.H., et al. Association between body composition, survival, and toxicity in advanced esophagogastric cancer patients receiving palliative chemotherapy. J. Cachexia. Sarcopenia Muscle 2019;10(1).

19. JÄRVINEN, T., ILONEN, I., KAUPPI, J., VOLMONEN, K., SALO, J., RÄSÄNEN, J. Low skeletal muscle mass in stented esophageal cancer predicts poor survival: A retrospective observational study. Thorac. Cancer 2018;9(11).

20. LEE, J.S., KIM, Y.S., KIM, E.Y., JIN, W. Prognostic significance of CT-determined sarcopenia in patients with advanced gastric cancer. PLoS One 2018;13(8).

21. LIN, J., ZHANG, W., HUANG, Y., CHEN, W., WU, R., CHEN, X., et al. Sarcopenia is associated with the neutrophil/ lymphocyte and platelet/lymphocyte ratios in operable gastric cancer patients: A prospective study. Cancer Manag. Res. 2018;10.

22. CESPEDES FEliCiAnO, E.M., AVRUTIN, E., CAAN, B.J., BOROIAN, A., MOURTZAKIS, M. Screening for low muscularity in colorectal cancer patients: a valid, clinic-friendly approach that predicts mortality. J. Cachexia. Sarcopenia Muscle 2018;9(5):898-908.

23. DOLAN, R.D., ALMASAUDI, A.S., DIEU, L.B., HORGAN, P.G., MCSORLEY, S.T., MCMILLAN, D.C. The relationship between computed tomography-derived body composition, systemic inflammatory response, and survival in patients undergoing surgery for colorectal cancer. J. Cachexia. Sarcopenia Muscle 2019;10(1):111-122.

24. XIAO, J., CAAN, B.J., CESPEDES FELICIANO, E.M., MEYERHARDT, J.A., KROENKE, C.H., BARACOS, V.E., et al. The association of medical and demographic characteristics with sarcopenia and low muscle radiodensity in patients with nonmetastatic colorectal cancer. Am. J. Clin. Nutr. 2019;109(3):615-625.

25. VASHI, P.G., GORSUCH, K., WAN, L., HILL, D., BLOCK, C., ID, D.G. Sarcopenia supersedes subjective global assessment as a predictor of survival in colorectal cancer 2019;69:1-14.

26. KURK, S., PEETERS, P., STELLATO, R., DORRESTEIJN, B., DE JONG, P., JOURDAN, M., et al. Skeletal muscle mass loss and dose-limiting toxicities in metastatic colorectal cancer patients. J. Cachexia. Sarcopenia Muscle 2019;10(4).

27. DOLAN, D.R., KNIGHT, K.A., MAGUIRE, S., MOUG, S.J. The relationship between sarcopenia and survival at 1 year in patients having elective colorectal cancer surgery. Tech. Coloproctol. 2019;23(9).

28. BROUGHMAN, J.R., WILliaMS, G.R., DEAL, A.M., YU, H., NYROP, K.A., ALSTON, S.M., et al. Prevalence of sarcopenia in older patients with colorectal cancer. J. Geriatr. Oncol. 2015;6(6).

29. CHOI, M.H., OH, S.N., LEE, I.K., OH, S.T., WON, D.D. Sarcopenia is negatively associated with long-term outcomes in locally advanced rectal cancer. J. Cachexia. Sarcopenia Muscle 2018;9(1).

30. LIEFFERS, J.R., BATHE, O.F., FASSBENDER, K., WINGET, M., BARACOS, V.E. Sarcopenia is associated with postoperative infection and delayed recovery from colorectal cancer resection surgery. Br. J. Cancer 2012;107(6).

31. RICHARDS, C.H., ROXBURGH, C.S.D., MACMILLAN, M.T., ISSWIASI, S., ROBERTSON, E.G., GUTHRIE, G.K., et al. The Relationships between Body Composition and the Systemic Inflammatory Response in Patients with Primary Operable Colorectal Cancer. PLoS One 2012;7(8):e41883.

32. TAKEDA, Y., AKIYOSHI, T., MATSUEDA, K., FUKUOKA, H., OGURA, A., MIKI, H., et al. Skeletal muscle loss is an independent negative prognostic factor in patients with advanced lower rectal cancer treated with neoadjuvant chemoradiotherapy. PLoS One 2018;13(4):e0195406. 
33. ONISHI, S., SHIRAKI, M., NISHIMURA, K., HANAI, T., MORIWAKI, H., SHIMIZU, M. Prevalence of sarcopenia and its relationship with nutritional state and quality of life in patients with digestive diseases. J. Nutr. Sci. Vitaminol. (Tokyo). 2018;64(6).

34. SAKAMOTO, T., YAGYU, T., UCHINAKA, E., MIYATANI, K., HANAKI, T., KIHARA, K., et al. Sarcopenia as a prognostic factor in patients with recurrent pancreatic cancer: A retrospective study. World J. Surg. Oncol. 2020;18(1).

35. NAUMANN, P., EBERlEIN, J., FARNIA, B., HACKERT, T., DEBUS, J., COMBS, S.E. Continued weight loss and sarcopenia predict poor outcomes in locally advanced pancreatic cancer treated with chemoradiation. Cancers (Basel). 2019;11(5).

36. GRUBER, E.S., JOMRICH, G., TAMANDL, D., GNANT, M., SCHINDL, M., SAHORA, K. Sarcopenia and sarcopenic obesity are independent adverse prognostic factors in resectable pancreatic ductal adenocarcinoma. PLoS One 2019;14(5).

37. AMRANI, M. EL, VERMERSCH, M., FUlBerT, M., PRODEAU, M., LECOLlE, K., HEBBAR, M., et al. Impact of sarcopenia on outcomes of patients undergoing pancreatectomy a retrospective analysis of 107 patients. Med. (United States) 2018;97(39).

38. VAN DIJK, D.P.J., BAKENS, M.J.A.M., COOLSEN, M.M.E., RENSEN, S.S., VAN DAM, R.M., BOURS, M.J.L., et al. Low skeletal muscle radiation attenuation and visceral adiposity are associated with overall survival and surgical site infections in patients with pancreatic cancer. J. Cachexia. Sarcopenia Muscle 2017;8(2).

39. NINOMIYA, G., FUJII, T., YAMADA, S., YABUSAKI, N., SUZUKI, K., IWATA, N., et al. Clinical impact of sarcopenia on prognosis in pancreatic ductal adenocarcinoma: A retrospective cohort study. Int. J. Surg. 2017;39.

40. PENG, P., HYDER, O., FIROOZMAND, A., KNEUERTZ, P., SCHULICK, R.D., HUANG, D., et al. Impact of Sarcopenia on Outcomes Following Resection of Pancreatic Adenocarcinoma. J. Gastrointest. Surg. 2012;16(8).

41. CHOI, Y., OH, D.-Y., KIM, T.-Y., LEE, K.-H., HAN, S.-W., IM, S.-A., et al. Skeletal Muscle Depletion Predicts the Prognosis of Patients with Advanced Pancreatic Cancer Undergoing Palliative Chemotherapy, Independent of Body Mass Index. PLoS One 2015;10(10):e0139749.

42. HA, Y., KIM, D., HAN, S., CHON, Y.E., LEE, Y. BIN, KIM, M.N., et al. Sarcopenia predicts prognosis in patients with newly diagnosed hepatocellular carcinoma, independent of tumor stage and liver function. Cancer Res. Treat. 2018;50(3).

43. BEGINI, P., GIGANTE, E., ANTONELLI, G., CARBONETTI, F., IANNICELLI, E., ANANIA, G., et al. Sarcopenia predicts reduced survival in patients with hepatocellular carcinoma at first diagnosis. Ann. Hepatol. 2017;16(1).

44. TAKAGI, K., YAGI, T., YOSHIDA, R., SHINOURA, S., UMEDA, Y., NOBUOKA, D., et al. Sarcopenia and American society of anesthesiologists physical status in the assessment of outcomes of hepatocellular carcinoma patients undergoing hepatectomy. Acta Med. Okayama 2016;70(5).

45. ZHOU, G., BAO, H., ZENG, Q., HU, W., ZHANG, Q. Sarcopenia as a prognostic factor in Hepatolithiasis-associated intrahepatic cholangiocarcinoma patients following hepatectomy: A retrospective study. Int. J. Clin. Exp. Med. 2015;8(10).

46. VAN VUGT, J.L.A., GASPERSZ, M.P., VUGTS, J., BUETTNER, S., LEVOLGER, S., DE BRUIN, R.W.F., et al. Low Skeletal Muscle Density Is Associated with Early Death in Patients with Perihilar Cholangiocarcinoma Regardless of Subsequent Treatment. Dig. Surg. 2019;36(2).

47. VAN VUGT, J.L.A., BUETTNER, S., LEVOLGER, S., COEBERGH VAN DEN BRAAK, R.R.J., SUKER, M., GASPERSZ, M.P., et al. Low skeletal muscle mass is associated with increased hospital expenditure in patients undergoing cancer surgery of the alimentary tract. PLoS One 2017;12(10).

48. CHOI, M.H., YOON, S.B., LEE, K., SONG, M., LEE, I.S., LEE, M.A., et al. Preoperative sarcopenia and post-operative accelerated muscle loss negatively impact survival after resection of pancreatic cancer. J. Cachexia. Sarcopenia Muscle 2018;9(2).

49. SIMONSEN, C., DE HEER, P., BJERRE, E.D., SUETTA, C., HOJMAN, P., PEDERSEN, B.K., et al. Sarcopenia and Postoperative Complication Risk in Gastrointestinal Surgical Oncology. Ann. Surg. 2018;268(1):58-69.

50. PANJE, C.M., HÖNG, L., HAYOZ, S., BARACOS, V.E., HERRMANN, E., GARCIA SCHÜLER, H., et al. Skeletal muscle mass correlates with increased toxicity during neoadjuvant radiochemotherapy in locally advanced esophageal cancer: A SAKK 75/08 substudy. Radiat. Oncol. 2019;14(1).

51. YAMAMOTO, K., NAGATSUMA, Y., FUKUDA, Y., HIRAO, M., NISHIKAWA, K., MIYAMOTO, A., et al. Effectiveness of a preoperative exercise and nutritional support program for elderly sarcopenic patients with gastric cancer. Gastric Cancer 2017;20(5).

52. VAN VUGT, J.L.A., LEVOlger, S., GHARBHARAN, A., KOEK, M., NIESSEN, W.J., BURGER, J.W.A., et al. A comparative study of software programmes for cross-sectional skeletal muscle and adipose tissue measurements on abdominal computed tomography scans of rectal cancer patients. J. Cachexia. Sarcopenia Muscle 2017;8(2).

Received $3^{\text {rd }}$ May 2021 